Hydroécol. Appl. (1991) Tome 3 Vol 2, pp. 209-240

\title{
Utilisation des mousses aquatiques comme bioindicateurs de contamination radioactive
}

\author{
J.P. Baudin, A. Lambrechts et M. Pally
}

IPSN/DPEI/SERE. Laboratoire de Radioécologie des Eaux Continentales. URSED CEN Cadarache, BP $N^{\circ} 113108$ Saint-Paul-Lez-Durance Cedex

Résumé. - II y a plus de 20 ans, la capacité de fixation des radionucléides par les bryophytes a été mise en évidence à la suite des essais nucléaires atmosphériques qui ont eu lieu notamment dans la période allant de 1957 à 1963. A partir de cette constatation, ces végétaux, et notamment les mousses, ont donné lieu à des études radioécologiques, leurs caractéristiques physiologique, biogéographique et écologique correspondant parfaitement aux critères auxquels doit répondre un bioindicateur de pollution. A cet égard, les travaux in situ menés depuis plusieurs années permettent de démontrer que les mousses aquatiques constituent de bien meilleurs indicateurs de contamination radioactive que le sédiment, les végétaux immergés et les poissons.

A partir du mois de juin 1986, les prélèvements d'échantillons de mousses ont permis de mettre en évidence les retombées radioactives dues à l'accident de Tchernobyl (présence de ${ }^{103} \mathrm{Ru},{ }^{106} \mathrm{Ru},{ }^{110 \mathrm{~m}} \mathrm{Ag},{ }^{134} \mathrm{Cs}$ et ${ }^{137} \mathrm{Cs}$ ). Le même type d'étude in situ permet de suivre l'évolution de la radioactivité dans les fleuves recevant les effluents liquides très faiblement radioactifs rejetés, après traitement, par les centrales électronucléaires. Dans ce domaine des résultats significatifs ont pu être rassemblés, en particulier pour certaines zones du Rhône et de la Seine (présence de ${ }^{54} \mathrm{Mn},{ }^{58} \mathrm{Co},{ }^{60} \mathrm{Co},{ }^{110 \mathrm{~m}} \mathrm{Ag},{ }^{134} \mathrm{Cs}$ et ${ }^{137} \mathrm{Cs}$ ). Dans certains cas une analyse détaillée des données permet d'établir des corrélations qualitatives et quantitatives entre la contamination des mousses et la composition des effluents liquides.

Malgré tout l'intérêt qu'elles présentent, les études in situ ne constituent qu'un constat ponctuel dans le temps. Pour une interprétation et une exploitation plus complète des résultats de ce type de travaux, comme pour reconstituer l'historique d'une pollution, des études expérimentales sont indispensables. Leur finalité est la connaissance des cinétiques d'accumulation et d'élimination ainsi que l'évaluation des facteurs de concentration et des périodes biologiques des radionucléides. Aussi de nombreuses expériences, dont la première date de 1968, ont été réalisées pour plusieurs radionucléides avec des bryophytes et en particulier deux espèces de mousse, Platyhypnidium riparioides et Fontinalis antipyretica. Une illustration de l'intérêt, et par conséquent, de la 
nécessité d'amplifier la complémentarité des études in situ et des travaux expérimentaux, est fournie par un exemple concret, fondé sur des mesures effectuées sur des mousses prélevées en aval du site de la centrale de Nogent-sur-Seine, en juillet 1989.

Mots clés. - radionucléides, mousses, eau douce, bioindicateur, études in situ, études expérimentales.

\begin{abstract}
The radionuclides retention ability of Bryophyta was shown up about twenty years ago, following the nuclear weapons tests, more particularly from 1957 to 1963. From that time onwards, these organisms, especially mosses, are the subjects of radioecological studies. Indeed, their physiological, biogeographical and ecological characteristics are perfectly adapted to the criterions, a biological indicator of pollution should have. Field studies, entered upon for several years in this connection, establish that aquatic mosses are better indicators or radioactive contamination than sediment, fishes and immersed plants.

Since June 1986, the radioactive fallout of the accident of Chernobyl, have been shown up by mosses samplings (detection of ${ }^{103} \mathrm{Ru},{ }^{106} \mathrm{Ru},{ }^{110 \mathrm{~m}} \mathrm{Ag},{ }^{134} \mathrm{Cs}$ et ${ }^{137} \mathrm{Cs}$ ). The evolution of radioactivity in the rivers, into which are released weakly radioactive liquid wastes from nuclear power plants, after they have been treated, is followed by the same kind of field studies. Thus, significant results could have been collected, particularly concerning some zones of the Rhône and the Seine where ${ }^{54} \mathrm{Mn},{ }^{58} \mathrm{Co},{ }^{60} \mathrm{Co},{ }^{110 m} \mathrm{Ag},{ }^{134} \mathrm{Cs}$ and ${ }^{137} \mathrm{Cs}$ have been found. Through some detailed analysis of the given informations, qualitive and quantitative correlations can be established between the contamination of mosses and the composition of liquid wastes.

Despite their interest, field studies are merely a punctual established fact in time. To have a more complete interpretation and handling of the data in this kind of work, as to give a chronological account of a pollution, experimental studies are absolutly necessary. They are used to study the kinetic of accumulation and elimination, as to estimate the concentrations factors and the biological half life of radionuclides. Therefore numerous experiments, the first one in 1968, have been undertaken with several radionuclides on Bryophyta and more precisely two species of mosses, Platyhypnidium riparioides and Fontinalis antipyretica. The interest, and consequently the necessity, to amplify the complementarity of field and experimental studies is illustrated by a concrete example, based on radioactivity measurements, carried out on mosses taken downstream the nuclear power station of Nogent-sur-Seine, in July 1989.
\end{abstract}

Key words. - Radionuclides, mosses, freshwater, bioindicator, field studies, experimental studies.

\section{1. - INTRODUCTION}

En radioécologie, comme dans d'autres domaines de l'étude de la pollution du milieu aquatique, est apparue la nécessité d'utiliser des bioindicateurs pouvant témoigner d'une contamination radioactive. En effet, compte tenu des niveaux très bas de radioac- tivité, le dosage des radionucléides dans l'eau soulève des problèmes de sensibilité difficiles à résoudre et ne permet pas, généralement, de déceler une pollution radioactive ou d'en mesurer l'ampleur véritable.

Les premières utilisations significatives des bryophytes comme bioindicateurs de pollution radioactive datent 
du début des années 1960. Elles concernaient des espèces terrestres et furent liées à la détection des retombées des nombreux essais nucléaires atmosphériques qui eurent lieu entre 1950 et 1963 (Bovard et Grauby, 1967; Svensson, 1967). Le constat des qualités des bryophytes terrestres, mises en évidence par ces études in situ, se traduisit très rapidement par la réalisation, dans notre laboratoire, de travaux radioécologiques expérimentaux destinés à tester les capacités de fixation des radionucléides par des espèces aquatiques, notamment des mousses. Depuis quelques années, parallèlement à la poursuite d'un programme expérimental, des travaux in situ se sont développés dans le cadre des études radioécologiques de l'environnement aquatique des sites d'implantation de certaines installations nucléaires et à la suite de l'accident de Tchernobyl (Baudin-Jaulent et Descamps, 1985; Descamps, 1987; Foulquier et al., 1987 ; Lambrechts et al., 1988; Descamps et Baudin-Jaulent, 1988). Ce type d'études s'applique en particulier aux centrales électronucléaires, dans l'environnement desquelles le laboratoire est très souvent intervenu à la demande d'E.D.F., pour effectuer des travaux de radioécologie aquatique. A cet égard, il faut souligner que la collaboration qui s'est développée et amplifiée ces dernières années avec la direction des différentes centrales et le Département Sécurité Radioprotection Environnement a permis une avancée qualitative importante de la connaissance des processus de contamination des mousses aquati- ques par les radionucléides. En effet, alors que pour les métaux lourds, par exemple, des interrogations demeurent quant aux rejets provenant de diverses industries, les données fournies par E.D.F. sur la composition et la radioactivité des effluents liquides des centrales électronucléaires permettent d'approfondir les connaissances en radioécologie, par une analyse plus poussée des résultats des travaux in situ. Ces renseignements représentent un apport indispensable pour valider les résultats des expériences de laboratoire qui sont irremplaçables pour évaluer les paramètres radioécologiques quantifiant les processus de transfert et qui constituent un complément nécessaire aux constats de terrain.

Dans cet article sont présentés quelques résultats tirés de l'ensemble des travaux menés sur les mousses dulçaquicoles par notre laboratoire depuis plusieurs années. Une première partie est consacrée à la mise en évidence de la qualité des mousses aquatiques comme indicateurs de contamination radioactive de différentes origines, fondée sur la comparaison de diverses données tirées d'études in situ. Dans la deuxième partie sont exposés plusieurs exemples de relation entre la composition des rejets liquides des centrales et la contamination des mousses. Quelques résultats expérimentaux sont rassemblés dans une troisième partie qui comporte également plusieurs exemples concrets mettant en évidence la complémentarité des études in situ et des travaux de laboratoire. 


\section{2. - MATÉRIEL ET MÉTHODES}

\subsection{Etudes in situ}

Au cours des études in situ, des échantillons des divers compartiments des écosystèmes considérés sont prélevés puis préparés en vue des mesures de radioactivité. Le sédiment est collecté au cône de Berthois. Les mousses sont prélevées à la main sur les roches ou les piles des ponts et les végétaux immergés sont cueillis à partir de la berge ou d'un bateau. Pour les poissons, la collecte s'effectue au filet, ou, le plus souvent, par pêche électrique.

Les échantillons sont rapidement transportés au laboratoire et placés en chambre froide à $4^{\circ} \mathrm{C}$ ou congelés (poissons). Leur conditionnement a essentiellement pour but de réduire à de petits volumes de grandes quantités de matière, afin de pouvoir mesurer de très faibles niveaux de radioactivité. Les sédiments sont séchés à $110^{\circ} \mathrm{C}$. Les végétaux sont très soigneusement lavés, séchés à $110^{\circ} \mathrm{C}$ puis incinérés à $560^{\circ} \mathrm{C}$. Les poissons subissent un traitement similaire.

Compte tenu des contraintes de quantité évoquées ci-dessus et pour ne pas épuiser les stations, les prélèvements de mousses sont volontairement limités si bien que l'échantillon considéré est représentatif d'une zone et non pas d'un point précis. En conséquence, les résultats concernant les mousses sont peu nombreux, voire uniques, pour chaque site étudié et il n'est pas possible d'effectuer de traitement statistique comme pour les autres compartiments de l'écosystème considéré (Foulquier et al., 1991).

\subsection{Etudes expérimentales}

Pour ces études, deux types de dispositifs sont utilisés, correspondant l'un à des conditions statiques et l'autre à des conditions dynamiques. Dans le premier cas, 50 à $100 \mathrm{~g}$ frais de mousses sont immergés dans un bac contenant 5 litres d'eau aérée par un diffuseur d'air et préalablement contaminée par l'ajout unique d'une solution de radionucléide. Ce protocole est peu satisfaisant car il est relativement éloigné des conditions de contamination in situ. Toutefois son utilisation est indispensable d'un point de vue expérimental car elle permet d'obtenir les premières données relatives au comportement du radionucléide étudié. Dans le deuxième cas, un apport continu de radionucléide est réalisé par l'utilisation d'une pompe péristaltique et les mousses sont soumises à un courant d'eau créé par une pompe centrifuge d'un débit de $300 \mathrm{I} . \mathrm{h}^{-1}$. Une variante de cette méthode consiste en une contamination intermittente, l'apport de radionucléide n'ayant lieu qu'un jour sur deux.

Au cours de la phase d'accumulation, la radioactivité de l'eau et des mousses est régulièrement mesurée. La concentration du radionucléide dans le milieu est évaluée sur des échantillons de $5 \mathrm{ml}$ d'eau brute et d'eau filtrée à $0,45 \mu \mathrm{m}$. Pour les mousses, 20 brins sont prélevés sur des tiges différentes de manière à 
échantillonner sur l'ensemble de l'étendue du bac. Ils sont rincés, séchés sur papier absorbant, passés à l'étuve à $100^{\circ} \mathrm{C}$ pendant 24 heures puis introduits dans des tubes de comptage. Lors de l'étude de l'élimination du radionucléide, le même protocole est utilisé, les mousses préalablement contaminées étant immergées dans de l'eau non radioactive, renouvelée toutes les 24 heures.

Les deux phases successives de l'expérimentation permettent d'évaluer deux paramètres radioécologiques essentiels. Le processus d'accumulation est quantifié par le facteur de concentration (FC) qui est le rapport, à chaque instant, entre la concentration du radionucléide dans l'organisme (Bq. $\left.{ }^{-1} \mathrm{sec}\right)$ et sa concentration dans l'eau (Bq. $\mathrm{ml}^{-1}$ ). L'étude de la décontamination conduit à l'évaluation de la période biologique (Tb) qui représente le temps nécessaire à un organisme pour éliminer la moitié du radionucléide préalablement fixé. La connaissance de ce paramètre permet de calculer la période effective du radionucléide (Te) qui est le temps après lequel un organisme a perdu la moitié de sa radioactivité initiale. Cette période effective se calcule seIon la formule $1 / T e=1 / \mathrm{Tb}+1 / \mathrm{Tp}$, Tp étant la période physique du radionucléide.

\section{3. - COMPARAISON DE DIFFÉRENTS INDICATEURS}

Cette comparaison porte sur les divers constituants des écosystèmes aquatiques dont des échantillons sont régulièrement prélevés au cours des études in situ: sédiments, poissons, végétaux supérieurs et mousses. Trois causes de la contamination du milieu ont été prises en compte : retombées d'essais nucléaires atmosphériques, retombées de l'accident de Tchernobyl et rejets liquides des centrales électronucléaires. Par rapport à la problématique exposée, il faut souligner que l'intérêt d'étudier ces trois cas réside dans le fait qu'ils diffèrent notamment par la nature et l'origine des radionucléides qui parviennent dans le milieu aquatique.

\subsection{Cas de retombées d'essais nucléaires atmosphériques}

En 1981, des prélèvements ont été effectués sur le site de la future centrale électronucléaire de Nogent-sur-Seine, dans le cadre du "point zéro" radioécologique aquatique habituellement réalisé avant la mise en route de ce type d'installation (Maubert et Descamps, 1983). D’après les résultats des mesures de la radioactivité gamma artificielle, il apparaît qu'un plus grand nombre de radionucléides est détecté dans les végétaux que dans les sédiments, et que les concentrations sont plus élevées chez les mousses (fontinales) que chez les myriophylles (fig. 1). Le ${ }^{137} \mathrm{Cs}$ provient des retombées des essais nucléaires atmosphériques réalisés entre 1950 et 1962 et les autres radionucléides trouvés sont dûs au tir effectué par la Chine le 16 octobre 1980. A propos de cet exemple, il faut souligner que 


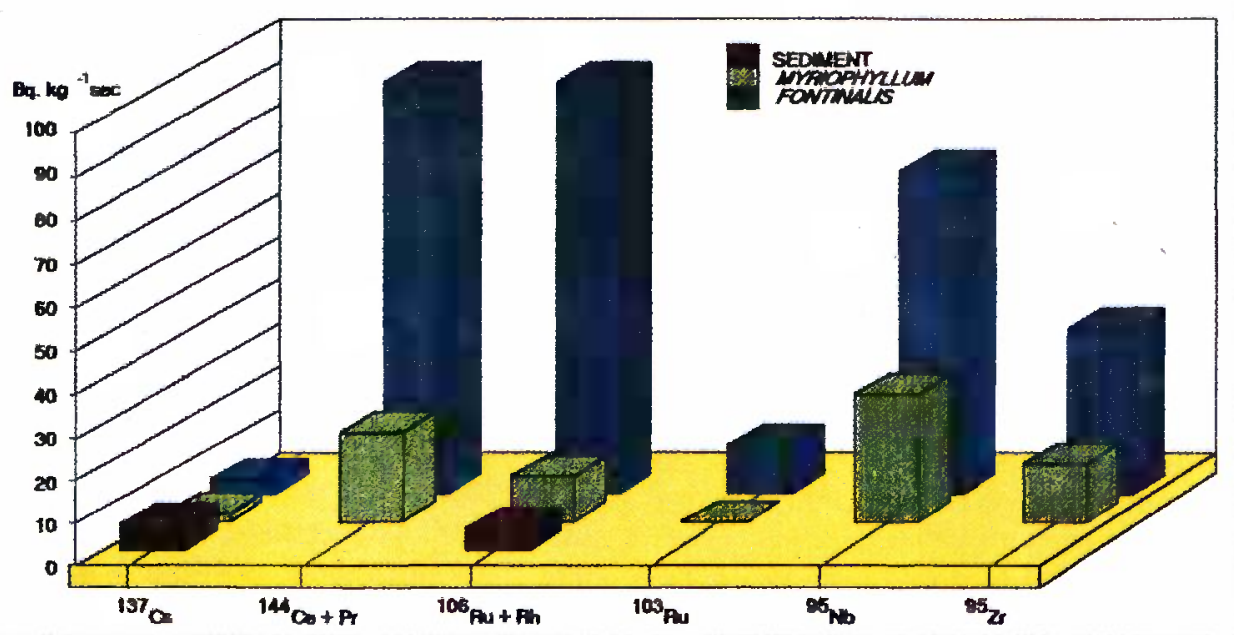

Fig. 1. - Comparaison de la qualité de différents indicateurs de l'impact radioécologique des retombées des essais nucléaires atmosphériques. Site de Nogent-sur-Seine, juillet 1981.

Fig. 1. - Efficiency comparison of different indicators of the radioecological impact of atmospheric weapon tests fallout. Site of Nogent-sur-Seine, July 1981.

les myriophylles constituent également de bons indicateurs, contrairement au sédiment qui pourtant est souvent considéré, de ce point de vue, comme un composant intéressant, dans la mesure où il est susceptible d'intégrer et de mémoriser les pollutions.

\subsection{Cas des retombées de l'acci- dent de Tchernobyl}

La deuxième situation envisagée concerne l'accident de la centrale de Tchernobyl, dont l'impact radiécologique a été étudié dans de nombreux écosystèmes aquatiques continentaux européens (Foulquier et Baudin-
Jaulent, 1990). Dans ce cas, il s'agit encore de retombées atmosphériques, mais dont l'origine est différente de celle traitée précédemment. En France, notre laboratoire a effectué un grand nombre d'observations, parmi lesquelles nous avons choisi d'exposer deux exemples concernant des zones non affectées par le rejet d'effluents liquides d'installations nucléaires.

Le premier cas est représenté par des mesures effectuées sur des échantillons prélevés dans la Garonne, sur le site de la centrale de Golfech, lors de la réalisation du "point zéro" radioécologique (Ottavi et Descamps, 1988). Cette étude s'est déroulée en juin 1986, c'est- 
à-dire peu de temps après l'accident qui est survenu le 26 avril 1986. On constate que les quatre radionucléides typiques des retombées de l'accident de Tchernobyl, c'est-àdire les césium 134 et 137 et les rhuténium 103 et 106, ne sont présents que chez les mousses. D'autre part, en ce qui concerne les radiocésium, les concentrations sont plus élevées, notamment par rapport à celles mesurées pour les poissons et le sédiment (fig. 2a). Comme précédemment, ce dernier apparaît comme un médiocre indicateur, par comparaison aux végétaux. Le rapport ${ }^{137} \mathrm{Cs} /{ }^{134} \mathrm{Cs}$ est de 2,6 pour le sédiment, 2,45 pour les végétaux supérieurs et 2,37 pour les mousses. Ces valeurs caractérisent bien les retombées de l'accident de Tchernobyl, le rapport isotopique étant de 2 à l'émission et augmentant au cours du temps en fonction des périodes physiques très différentes des deux isotopes

$$
\left({ }^{137} \mathrm{Cs} /{ }^{134} \mathrm{Cs}=2 \mathrm{e}^{-0,69(1 / 30-1 / 2,06) t}\right) .
$$

Le deuxième cas a trait à un petit fleuve de la côte orientale de la Corse, le Tavignano, dont l'embouchure se trouve près d'Aléria, à $60 \mathrm{~km}$ environ au sud de Bastia (Descamps et Baudin-Jaulent, 1988). Cet exemple démontre encore la très nette supériorité des mousses comme indicateurs de contamination radioactive, aussi bien par le nombre des radionucléides trouvés que par leur concentration (fig. 2b). Par rapport au cas précédent, il faut noter la présence de $I^{1110 \mathrm{~m}} \mathrm{Ag}$, radioélément éga- lement caractéristique des retombées de l'accident de Tchernobyl mais qui n'est pas toujours détecté. Une autre constatation doit être soulignée; elle concerne les truites pour lesquelles les concentrations en ${ }^{134} \mathrm{Cs},{ }^{137} \mathrm{Cs}$ et ${ }^{110 \mathrm{~m}} \mathrm{Ag}$ sont aussi élevées que pour les mousses. Ce fait, a priori surprenant, peut s'expliquer par une décontamination partielle des mousses, 3 mois après l'accident et, inversement, une accumulation des radionucléides par ingestion de proies contaminées chez la truite. Cette hypothèse, avancée pour expliquer ce constat in situ, s'appuie sur des travaux expérimentaux, dont les résultats indiquent clairement des possibilités de transfert et de bioaccumulation, à partir de la nourriture, du ${ }^{137} \mathrm{Cs}$ et du ${ }^{110 \mathrm{~m}} \mathrm{Ag}$ par des poissons dulçaquicoles (Baudin, 1970; Lambrechts, 1984; Garnier-Laplace, 1991). Le rapport entre les deux isotopes du césium est de 2,57 pour le sédiment, 2 pour les végétaux immergés, 2,47 pour les mousses et 2,1 pour les truites, valeurs globalement conformes à celle correspondant aux retombées, trois mois après l'accident, qui est de 2,16.

\subsection{Cas des effluents liquides des centrales électronucléaires}

Compte tenu des études menées sur l'ensemble des sites d'implantation des centrales électronucléaires, les données relatives à l'impact radioécologique des effluents liquides sont très nombreuses et un choix a donc été 

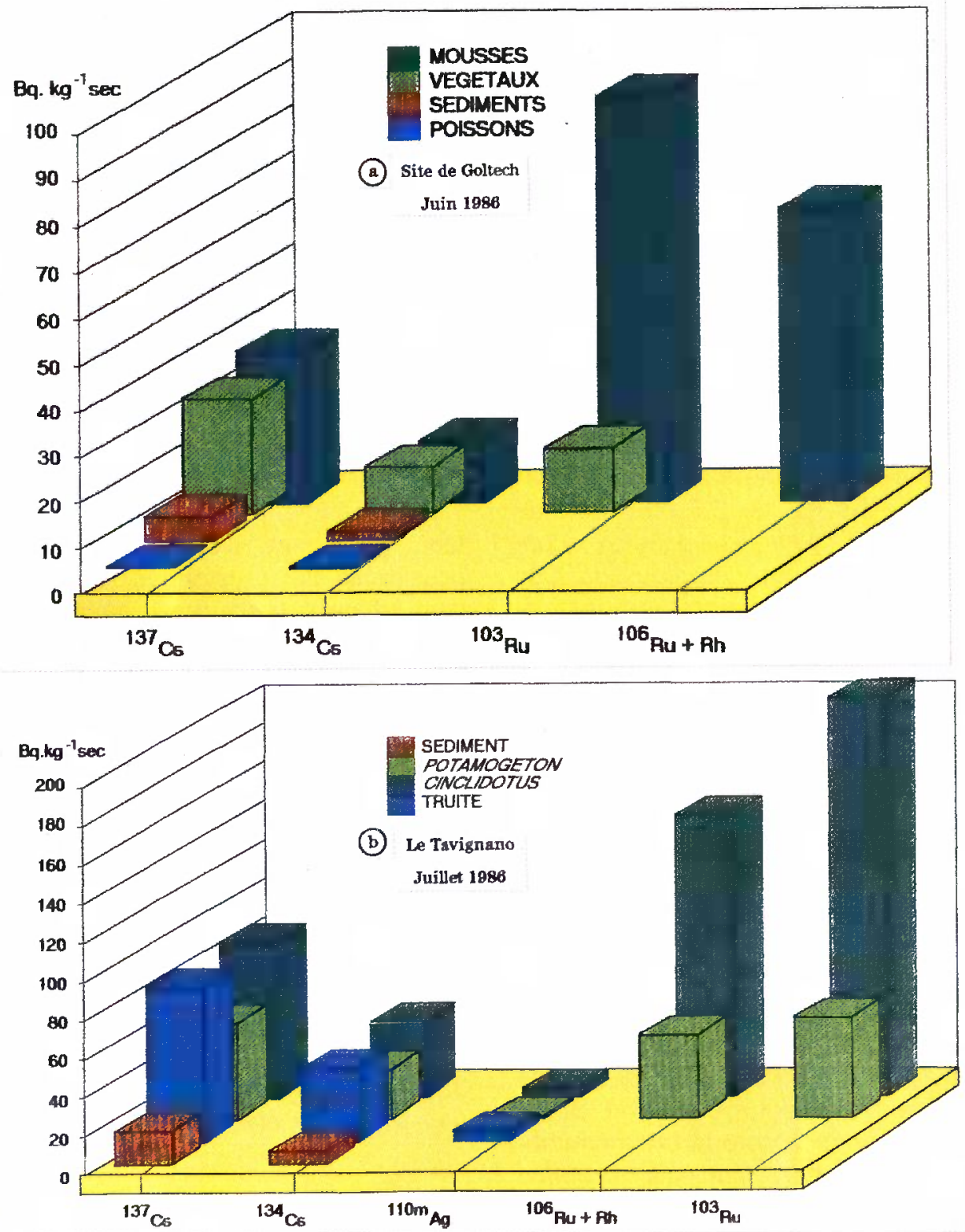

Fig. 2. - Comparaison de la qualité de différents indicateurs de l'impact radioécologique des retombées atmosphériques provenant de l'accident de Tchernobyl.

Fig. 2. - Efficiency comparison of different indicators of the radioecological impact of Chernobyl accident fallout. 
nécessaire. Deux exemples, fondés sur des études réalisées en juillet 1989 , ont été retenus : le site du Bugey où se trouve la plus ancienne centrale construite sur le Rhône et celui de Nogent-sur-Seine, en raison de la réalisation d'une étude particulière dont nous ferons état à plusieurs reprises.

A $4 \mathrm{~km}$ en aval du Centre de Production Nucléaire du Bugey, on constate que 6 radionucléides ont pu être mesurés dans les mousses (Cinclidotus riparius) alors que seulement 5 apparaissent dans le sédiment et à des concentrations de $10\left({ }^{134} \mathrm{Cs}\right.$ et $\left.{ }^{137} \mathrm{Cs}\right)$ à 60 fois $\left({ }^{110 \mathrm{~m}} \mathrm{Ag}\right)$ inférieures (fig. 3a). Contrairement aux cas précédents, les poissons ne présentent qu'une très faible contamination, par comparaison aux autres compartiments. Par exemple, la concentration en ${ }^{137} \mathrm{Cs}$ est 40 fois supérieure pour Cinclidotus riparius que pour les poissons alors que dans le cas des retombées de Tchernobyl elle n'était que de 12 fois plus élevée (site de Golfech) ou même tout à fait similaire (le Tavignano). Au-delà de la très forte capacité d'accumulation des radionucléides par les mousses, une fois encore mise en évidence, les différences notables relevées pour les poissons tendent à indiquer que le comportement et la biodisponibilité du césium varie assez nettement en fonction des caractéristiques des écosystèmes.

En ce qui concerne le site de Nogent-sur-Seine, les études menées au début du mois de juillet 1989 ont permis de rassembler diverses données radioécologiques d'un grand intérêt. Pour la campagne de prélèvements, qui a concerné le sédiment, des végétaux immergés et des mousses, 5 stations, échelonnées le long du fleuve entre 2 et $100 \mathrm{~km}$ en aval de l'installation, ont été prises en compte. Une autre caractéristique de ces travaux est liée aux mesures de la radioactivité des mousses qui, dans un premier temps, ont été effectuées sur des échantillons frais, quelques heures seulement après leur prélèvement. Les résultats obtenus, confirment la très nette supériorité des mousses sur les sédiments et les végétaux immergés comme indicateurs de pollution radioactive (fig. $3 b$ ). Ainsi, dans les fontinales prélevées $2 \mathrm{~km}$ en aval de la centrale, le nombre de radionucléides détectés est de 11 alors qu'il n'est que de 6 dans le sédiment et 5 dans les potamots. D'autre part, pour les éléments communs, les concentrations sont très largement supérieures chez les mousses, sauf pour les deux isotopes du césium dont la présence dans les écosystèmes aquatiques pose, depuis l'accident de Tchernobyl, un problème particulier qui sera traité ultérieurement. A propos de ces résultats, il nous paraît intéressant de souligner le grand nombre d'éléments mesurés, ce qui s'explique par le protocole opératoire suivi, les échantillons ayant été mesurés frais quelques heures seulement après leur prélèvement. Ainsi, la détection de l'iode est liée à l'absence de traitement, et notamment de dessiccation à l'étuve et d'incinération. II faut également mentionner la présence du tellure $123 \mathrm{~m}$, qui est un 

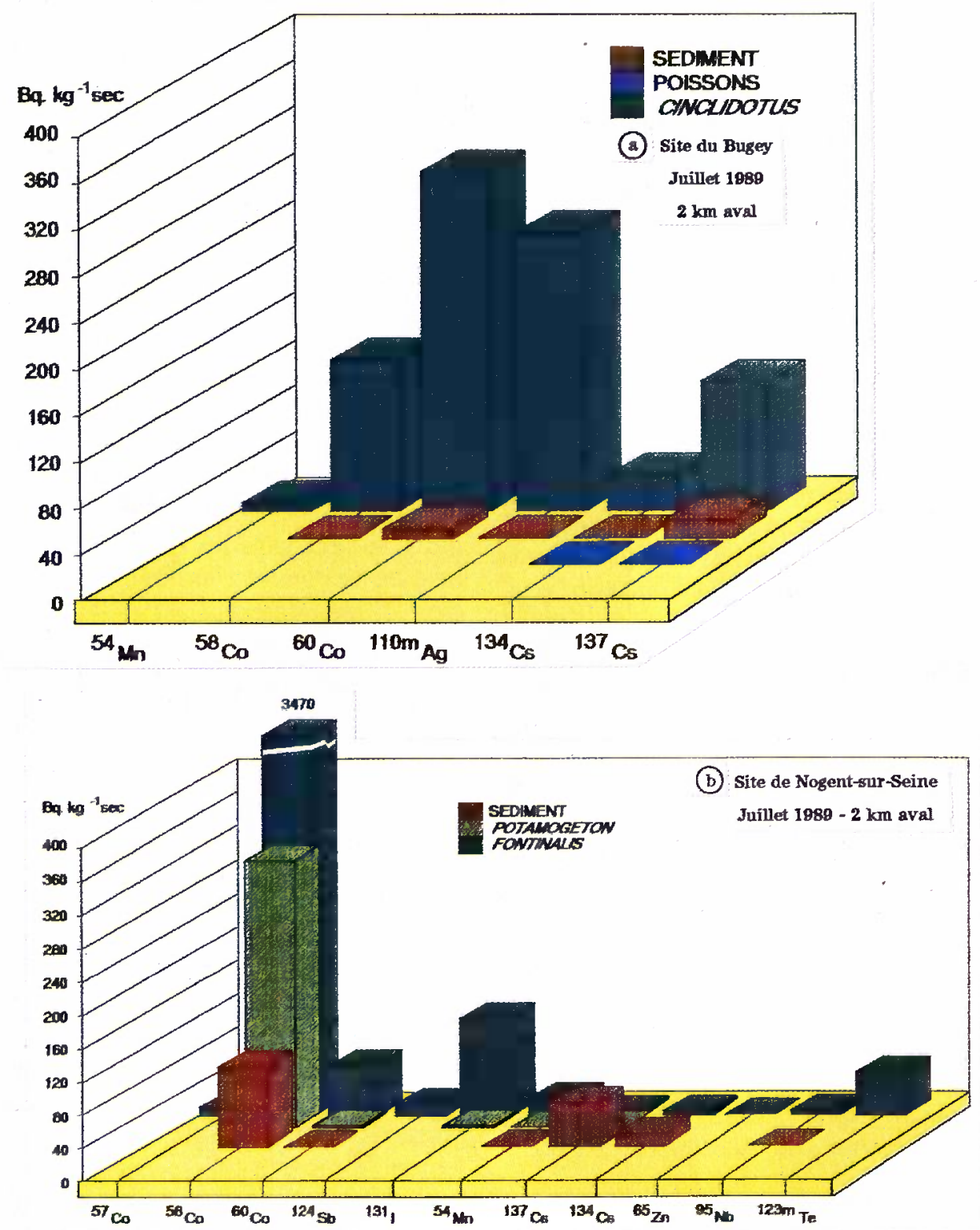

Fig. 3. - Comparaison de la qualité de différents indicateurs de l'impact radioécologique des effluents liquides des centrales nucléaires.

Fig. 3. - Efficiency comparison of different indicators of the radioecological impact of liquid wastes from nuclear power plants. 
produit de fission direct, père de $\left.\right|^{131} \mid$, et qui n'avait jamais pu être mis en évidence dans des échantillons prélevés in situ, même après l'accident de Tchernobyl alors qu'il était donné dans l'inventaire des produits rejetés dans l'atmosphère.

Si l'on considère les données relatives aux prélèvements effectués $4 \mathrm{~km}$ en aval de la centrale, la qualité de bioindicateur des mousses est encore plus probante. Ainsi, 10 radionucléides sont encore mesurés dans les fontinales (le ${ }^{65} \mathrm{Zn}$ a disparu) et seulement 2 dans les potamots. Cette comparaison constitue une transition vers le problème de l'évaluation de l'influence de la distance du point de rejet des effluents sur la contamination des mousses. En juillet 1989, des échantillons de la même espèce de mousse (Fontinalis antipyretica) ont été prélevés le même jour dans 3 stations situées à 2, 4 et $8 \mathrm{~km}$ en aval du Centre de Production Nucléaire de Nogent-sur-Seine. Les résultats montrent que le nombre de radionucléides détectés et leur concentration diminue en fonction de la distance (fig. 4a). Toutefois, la présence du ${ }^{54} \mathrm{Mn}$ et des 3 isotopes du cobalt est très nettement marquée après $8 \mathrm{~km}$, ce qui témoigne d'une forte affinité pour les mousses et de leur disponibilité au sein de la masse d'eau, malgré la distance. Pour le site du Bugey, une comparaison a pu être établie avec l'espèce Cinclidotus riparius (fig. 3b) entre deux stations, situées à 4 et $12 \mathrm{~km}$ en aval du rejet de la centrale. Contrairement aux constatations précédentes, tous les radionucléides présents dans les mousses au point
$4 \mathrm{~km}$ aval le sont encore $8 \mathrm{~km}$ plus loin, alors que pour la Seine la moitié avait disparu en $6 \mathrm{~km}$. Par contre, en ce qui concerne les concentrations du ${ }^{54} \mathrm{Mn}$, du ${ }^{58} \mathrm{Co}$, du ${ }^{60} \mathrm{Co}$ et du ${ }^{137} \mathrm{Cs}$, la diminution est plus importante dans le cas du Bugey. Pour le ${ }^{58} \mathrm{Co}$, par exemple, la concentration dans les mousses diminue de $40 \%$ en $6 \mathrm{~km}$ dans la Seine et de $80 \%$ en $8 \mathrm{~km}$ dans le Rhône. Ces différences sont probablement liées à une affinité particulière des mousses pour les divers radionucléides et aux caractéristiques hydrologiques des deux fleuves, notamment à leur débit au moment de l'émission des effluents liquides. Mais au-delà de l'aspect quantitatif de ces deux exemples, le point essentiel qui doit être souligné est le fait que les mousses permettent de mettre en évidence certains radionucléides, plusieurs kilomètres en aval de leur rejet, en les concentrant à des niveaux significatifs alors qu'ils ne sont pas, ou très difficilement, détectés dans l'eau.

\section{4. - RELATION ENTRE LA COMPOSITION DES EFFLUENTS LIQUIDES DES CENTRALES ET LA CONTAMINATION DES MOUSSES}

En commençant ce paragraphe, il est nécessaire de bien préciser que les prélèvements de mousses ont généralement lieu une seule fois par an, au cours des mois d'été, alors que les indications que nous possédons sur les rejets portent sur l'année entière et concernent uniquement les 8 principaux radionucléides identifiés (hors 

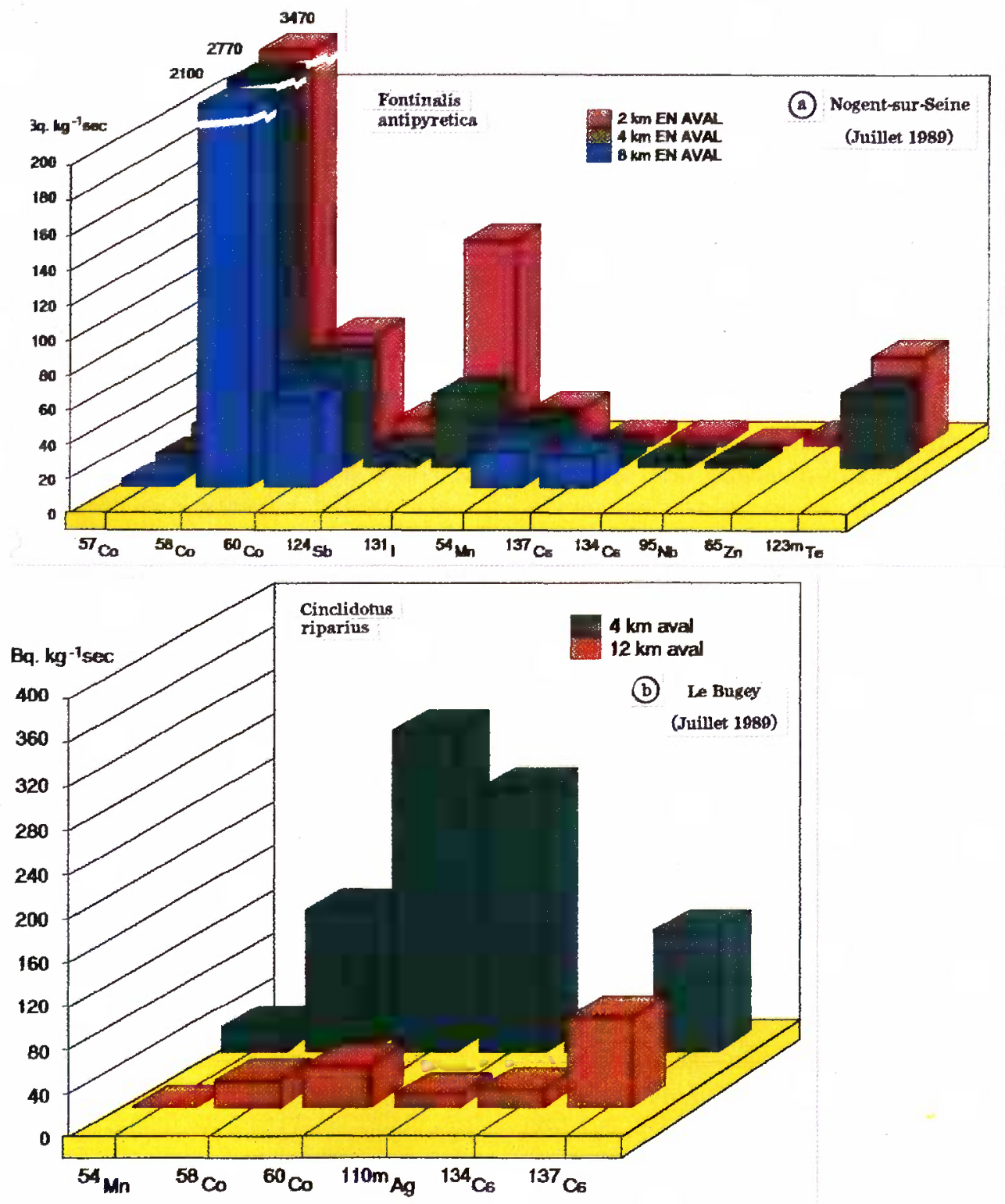

Fig. 4. - Influence de la distance du point de rejet des effluents liquides sur la contamination des mousses.

Fig. 4. - Influence of the distance of liquid wastes discharge point on the mosses contamination. 
tritium). Dans ces conditions, il est totalement impossible d'établir des corrélations strictes entre la composition des effluents et l'importance relative et la concentration des radionucléides dans les mousses. Toutefois, malgré les approximations qu'elle implique, cette démarche constitue une première approche qui a permis de dégager quelques données radioécologiques dont lintérêt justifie la poursuite de ce type d'études. La plupart des travaux in situ étant consacrée au Rhône, les résultats présentés portent essentiellement sur les sites des centrales implantées sur ce fleuve, à l'exception de celle de Nogent-sur-Seine.

\subsection{Site du Bugey}

Compte tenu de la continuité des études menées sur ce site, les données exposées représentent la moyenne sur 3 ans (1987-1989) de la composition des effluents liquides et de la concentration des radionucléides dans les mousses (Cinclidotus riparius) prélevées $4 \mathrm{~km}$ en aval de la centrale. La composition des effluents prise en compte est celle de l'ensemble de l'installation, c'est-àdire les 4 réacteurs à eau sous pression et le réacteur à uranium naturel-graphite-gaz (EDF, 1987, 1988 , 1989). La comparaison des deux groupes de valeurs (fig. 5a) montre qu'il y a une relation qualitative et partiellement quantitative entre la contamination des mousses et la composition des effluents. Ainsi, dans les mousses, 6 des 8 radionucléides identifiés dans les rejets sont détectés et la concentration la plus élevée est celle du ${ }^{58} \mathrm{Co}$ qui est l'élément le plus abondant des effluents. Toutefois plusieurs distorsions apparaissent, notamment l'absence de $\left|{ }^{\prime 131}\right|$ et de $\mathrm{l}^{124} \mathrm{Sb}$ dans les mousses. Pour le premier cette absence est tout à fait normale en raison de sa très courte période physique ( 8 jours) et du traitement des échantillons (dessiccation et incinération). Pour le second, par contre, les raisons précédentes ne sont pas valables. En effet l'antimoine n'est pas volatil comme l'iode et sa période physique est de 60 jours. D'autre part il représente $22 \%$ de la radioactivité totale (hors tritium) des effluents et devrait donc être retrouvé dans les mousses, compte tenu de la fréquence des rejets.

Abstraction faite de $\left|{ }^{131}\right|$ et de P'124Sb, les concentrations des radionucléides dans les mousses sont globalement proportionnelles aux quantités rejetées. Cependant, le calcul du rapport entre les deux types de données (Bq. $\mathrm{kg}^{-1} \mathrm{sec}$ de mousses/ GBq dans effluents) permet de mieux estimer les capacités de fixation des divers éléments par les mousses. $\mathrm{Ce}$ rapport est de 11,7 pour le ${ }^{54} \mathrm{Mn}, 8,1$ pour le ${ }^{58} \mathrm{Co}, 6$ pour le ${ }^{60} \mathrm{Co}, 11,5$ pour

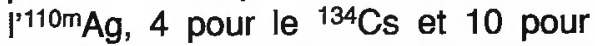
le ${ }^{137} \mathrm{Cs}$. La hiérarchie de ces valeurs est significativement différente de celle concernant les concentrations et met en évidence une fixation préférentielle du ${ }^{54} \mathrm{Mn}$ et de $\mathrm{I}^{1110 \mathrm{~m}} \mathrm{Ag}$. Toutefois, cette constatation n'a qu'une valeur relative et mérite d'être confir- 
mée, étant donné que, si des paramètres biotiques peuvent influer sur l'accumulation et la désorption des différents radionucléides, il n'est pas exclu que la composition des effluents rejetés dans les jours précédant le prélèvement des mousses intervienne fortement dans le résultat des mesures. A cet égard le cobalt permet d'étayer cette éventualité dans la mesure où le rapport est différent pour les deux isotopes $\left(8,1\right.$ pour le ${ }^{58} \mathrm{Co}$ et 6 pour le ${ }^{60} \mathrm{Co}$ ). Cette discrimination n'a pas de fondement physiologique ni chimique et ne peut s'expliquer que par le fait que le rejet le plus récent contenait proportionnellement plus de ${ }^{58} \mathrm{Co}$ que de ${ }^{60} \mathrm{Co}$, par rapport à la moyenne des rejets annuels. Le même problème se pose pour le césium, le rapport Bq. $\mathrm{kg}^{-1} \mathrm{sec}$ de mousse/GBq total rejetés étant de 4 pour le ${ }^{134} \mathrm{Cs}$ et de 10 pour le ${ }^{137} \mathrm{Cs}$. L'explication précédente pourrait être avancée pour justifier cette différence, mais le rapport isotopique ${ }^{137} \mathrm{Cs} /{ }^{134} \mathrm{Cs}$ est de 1,36 dans les effluents $(15,5 / 11,4)$ et de 3,40 dans les mousses (156/46) ce qui est tout à fait anormal. En fait, cette anomalie apparente est liée aux retombées de l'accident de Tchernobyl, qui sont caractérisées, comme cela a été évoqué précédemment, par un rapport isotopique des deux césium dont il est possible de calculer l'évolution en fonction du temps. Toutefois, dans le cas présenté, l'importance de l'impact des retombées de l'accident de Tchernobyl par rapport aux rejets du réacteur UNGG ne peut être mis clairement en évidence, les données représentant des moyennes établies sur 3 ans.

\subsection{Site de Saint-Alban}

Pour ce cite, les données concernent l'espèce Fontinalis antipyretica dont des échantillons ont été prélevés à la fin du mois d'août $1988,70 \mathrm{~km}$ en aval du point de rejet des effluents liquides. Les résultats confirment tout à fait la qualité des mousses comme bioindicateurs de pollution radioactive, puisque la concentration de 6 des 8 radionucléides principaux a pu être évaluée, malgré le grand éloignement de la station de prélèvement par rapport au point de rejet (fig. 5b). Globalement, les caractéristiques dégagées pour le site du Bugey se retrouvent dans ce cas : concentration la plus forte pour le ${ }^{58} \mathrm{Co}$ qui est le composant majeur des effluents, absence de l'124Sb qui représente pourtant $15 \%$ de la radioactivité hors tritium des effluents et fixation préférentielle du ${ }^{54} \mathrm{Mn}$ mise en évidence par les valeurs du rapport [radionucléide] $]_{\text {mousse/quantité totale rejetée. }}$ Par contre, à l'inverse des résultats relatifs aux mousses prélevées sur le site du Bugey, ce rapport est nettement moins élevé pour $1^{1} 110 \mathrm{~m} \mathrm{Ag}(0,4)$ que pour le ${ }^{58} \mathrm{Co}(6,5)$ et le ${ }^{60} \mathrm{Co}(5,7)$. Etant donné que les mousses proviennent d'une station située $70 \mathrm{~km}$ en aval de la centrale de Saint-Alban, cette constatation est conforme aux résultats de l'étude de l'influence de la distance du point de rejet des effluents sur la contamination de Cinclidotus riparius, fondée sur des prélèvements effectués sur le site de la centrale du Bugey. En effet, cette étude ( $\$ 3.3$ et fig. 4b) a montré que l'accroissement de la distance se tra- 
duisait dans les mousses par une diminution de la concentration de tous les radionucléides, diminution nettement plus marquée pour $1{ }^{1110 \mathrm{~m}} \mathrm{Ag}$ $(95 \%)$ que pour le ${ }^{58} \mathrm{Co}(79 \%)$ et le ${ }^{60} \mathrm{Co}(87 \%)$.

Les deux isotopes du césium posent un problème particulier qu'il convient de souligner. En effet, alors que les quantités rejetées sont les plus faibles, leur concentration dans les mousses (fig. 3b), se traduit par un rapport [Cs] mousses/quantité de Cs rejeté dont les valeurs sont largement supérieures à celles calculées pour les autres radionucléides. D'autre part, ce rapport est très différent pour les deux isotopes (39 pour le ${ }^{134} \mathrm{Cs}$ et 207 pour le ${ }^{137} \mathrm{Cs}$ ) alors qu'il n'y a aucune justification écologique ou physiologique à une telle discrimination. En fait ces distorsions peuvent s'expliquer par l'impact des retombées de l'accident de Tchernobyl, dont l'une des conséquences est la présence durable des radiocésium dans les milieux aquatiques. Ainsi le rapport isotopique ${ }^{137} \mathrm{Cs} /{ }^{134} \mathrm{Cs}$ qui est de 5,3 dans les mousses est fortement déterminé par la valeur de celui caractérísant les retombées et qui était d'environ 4 au moment du prélèvement des mousses (août 1988).

\subsection{Site de Cruas}

Pour ce site, la contamination des mousses présente les caractères généraux définis précédemment et correspond, abstraction faite de |'131| et $\left.\right|^{\prime 124} \mathrm{Sb}$, à la composition des effluents (fig. $5 \mathrm{c}$ ). A cet égard, il faut souligner la présence constante des radiocobalt dans les mousses et la corrélation entre la concentration en $110 \mathrm{~m} A g$ et son importance dans les rejets. En ce qui concerne les radiocésium, la part incombant aux retombées de l'accident de Tchernobyl dans la contamination des mousses apparait ici très clairement. Ainsi, le rapport isotopique ${ }^{137} \mathrm{Cs} /{ }^{134} \mathrm{Cs}$ n'est que de 1,70 dans les effluents alors qu'il est de 2,78 dans les mousses, cette deuxième valeur étant très proche de celle calculée pour les retombées à la date des prélèvements (juin 1987) et qui est de 2,70.

\subsection{Site de Nogent-sur-Seíne}

Certains aspects des résultats concernant ce site ont déjà été évoqués antérieurement (\$3.3.), et nous rappellerons que la concentration de 11 radionucléides a pu être évaluée dans les fontinales prélevées $2 \mathrm{~km}$ en aval de la centrale (fig. 3b). En ne considérant que les 8 principaux répertoriés dans les effluents (fig. 6), on constate que tous sont présents, à l'exception de l'110m Ag qui est rejeté en trop faible quantité (moins de $0,1 \mathrm{GBq}$ pour l'année 1989) pour être détecté dans les organismes vivants (fig. 4). Les 4 autres radionucléides, qui représentent une radioactivité de $72 \mathrm{~Bq} . \mathrm{kg}^{-1} \mathrm{sec}$, sont le ${ }^{57} \mathrm{Co}$, le ${ }^{65} \mathrm{Zn}$, le ${ }^{95} \mathrm{Nb}$ et le ${ }^{123 \mathrm{~m}} \mathrm{Te}$, ce dernier représentant $52 \mathrm{~Bq} \cdot \mathrm{kg}^{-1} \mathrm{sec}$. Une fois encore, il faut souligner la très grande prépondérance du ${ }^{58} \mathrm{Co}$, dont la proportion est plus élevée dans les 

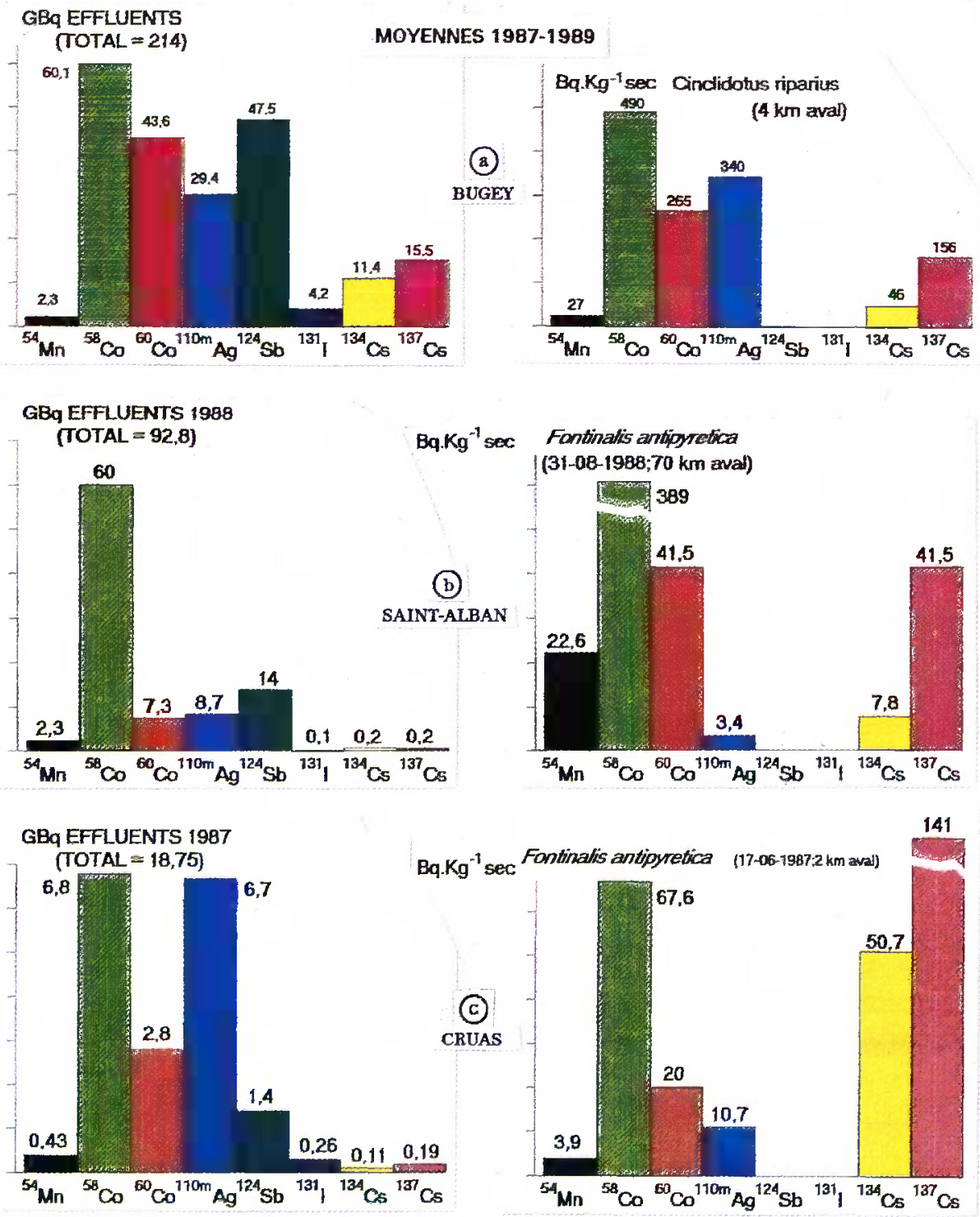

Fig. 5. - Exemple de relation entre la composition des effluents liquides des centrales nucléaires et la contamination radioactive des mousses dulçaquicoles.

Fig. 5. - Exemple of relation between the composition of liquid wastes from nuclear power plants and the radioactive contamination of freshwater mosses. 
mousses $(91,9 \%)$ que dans les effluents $(67 \%)$. Au sujet de l'estimation de la capacité de fixation des radionucléides, le rapport entre leur concentration dans les mousses (Bq. $\left.\mathrm{kg}^{-1} \mathrm{sec}\right)$ et la quantité rejetée dans l'année (GBq) est de 91 pour le ${ }^{58} \mathrm{Co}, 79$ pour le ${ }^{60} \mathrm{Co}$ et 46 pour le ${ }^{54} \mathrm{Mn}$. La hiérarchie de ces valeurs valide la plupart des données obtenues, dans des conditions moins rigoureuses, pour les autres sites.

La présence de $\left.\right|^{13131} \mid$ et de $\left.\right|^{1124} \mathrm{Sb}$ dans les mousses a été particulièrement soulignée étant donné que c'est la seule fois qu'ils ont pu être mis en évidence en aval d'une centrale nucléaire. En plus de cette originalité, due, rappelons-le, à la procédure utilisée pour mesurer la radioactivité des échantillons, l'analyse des résultats apporte d'intéressantes données concernant ces deux radionucléides, dont le comportement est tout à fait différent. Ainsi, l'131| représente seulement $1 \%$ de la radioactivité des effluents et $3,1 \%$ de celle des mousses, alors que pour $\left.\right|^{124} \mathrm{Sb}$ ces proportions sont respectivement de $20,3 \%$ et $0,4 \%$. En terme de capacité de fixation cela se traduit par un rapport [radionucléide] $]_{\text {mousses }} / \mathrm{GBq}$ rejetés de 196 pour |'131।, valeur deux fois plus élevée que celle relative au ${ }^{58} \mathrm{Co}$. A l'inverse le rapport n'est que de 1,4 pour $l^{1} 24 \mathrm{Sb}$, ce qui explique le fait qu'il ne soit pas détecté et suggère que le facteur de concentration de ce radionucléide par les bryophytes doit être beaucoup moins élevé, que celui des radiocolbalt, de $\mathrm{I}^{1110 \mathrm{~m}} \mathrm{Ag}$, du ${ }^{54} \mathrm{Mn}$ et de $|131|$.

\section{5. - ÉTUDES EXPÉRIMENTALES}

Les expériences menées depuis de nombreuses années sur les bryophytes dulçaquicoles, dans le Laboratoire de Radioécologie des Eaux Continentales, ont fait l'objet de plusieurs publications (Grauby et Foulquier, 1967; Hébrard et al., 1968; Hébrard et Foulquier, 1975; Foulquier et Hébrard, 1976; Maurel-Kermarrec, 1984 ; Maurel-Kermarrec et al., 1983, 1985). Dans ce paragraphe, seuls quelques résultats non encore publiés sont succinctement présentés ainsi qu'une synthèse des données relatives à la contamination de Platyhypnidium riparioides par plusieurs radionucléides.

Les expériences les plus récentes ont été réalisées avec $1^{110 m} \mathrm{Ag}, \mathrm{l}^{125} \mathrm{Sb}$ et le ${ }^{106} \mathrm{Ru}$, pour lesquels les connaissances sur les transferts en eau douce sont encore très limitées. Outre ces lacunes, ces trois radionucléides ont été retenus en raison de leur importante proportion dans les effluents liquides des centrales électronucléaires $\left({ }^{110 \mathrm{~m}} \mathrm{Ag}\right.$ et $\left.{ }^{125} \mathrm{Sb}\right)$ ou des usines de retraitement des combustibles irradiés ( $\left.{ }^{106} \mathrm{Ru}\right)$. Pour l'antimoine, il faut préciser que c'est l'isotope ${ }^{124} \mathrm{Sb}$ qui se trouve dans les effluents liquides, mais que $\mathrm{I}^{125} \mathrm{Sb}$ a été préféré pour les études in vitro en raison de sa période physique plus longue qui facilite l'expérimentation.

Généralement, la durée des expériences avec les mousses n'excède pas une semaine. Cette durée relativement courte est due au fait qu'en 

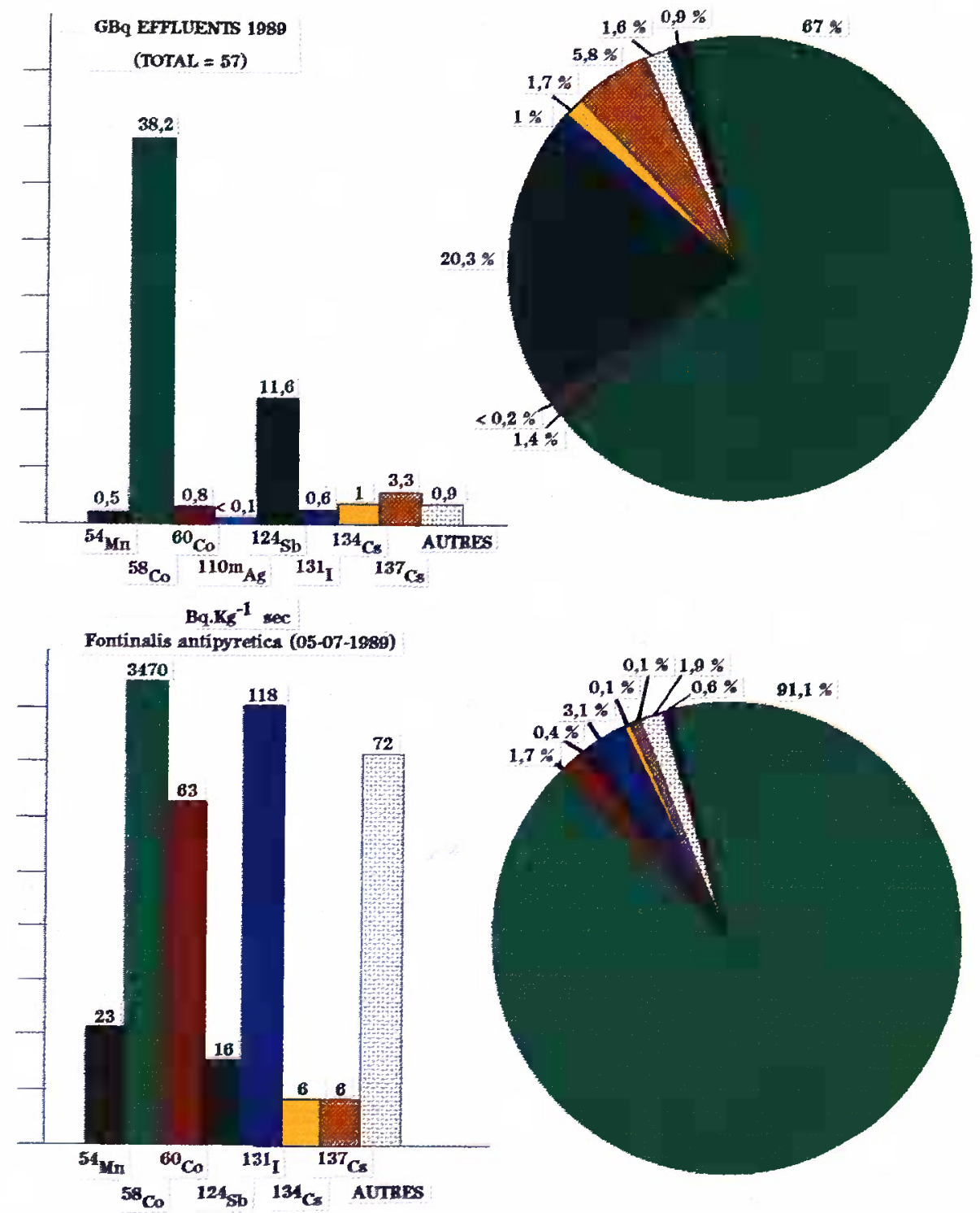

Fig. 6. - Relation entre la composition des effluents liquides de la centrale de Nogent-sur-Seine et la contamination radioactive des mousses prélevées à $2 \mathrm{~km}$ en aval du point de rejet.

Fig. 6. - Relation between the composition of liquid wastes from the nuclear power plant of Nogent-sur-Seine and the radioactive contamination of mosses sampled $2 \mathrm{~km}$ downstream the discharge point. 
conditions statiques, un épuisement du radionucléide dans le milieu se produit rapidement dans la plupart des cas et qu'en conditions dynamiques, la radioactivité totale pouvant être mise en jeu est limitée pour des raisons de sécurité liée à la radioprotection des expérimentateurs.

\subsection{Données sur la contamina- tion des mousses par $\mathbf{1}^{110 \mathrm{~m}} \mathbf{A g}$, li125 $^{12 b}$ et le ${ }^{106} \mathrm{Ru}$}

\subsection{1. $L^{, 110 m} \mathrm{Ag}$}

Les expériences menées avec ['110m Ag illustrent parfaitement les problèmes évoqués ci-dessus. Ainsi, en conditions statiques, après $24 \mathrm{~h}$ seulement, la radioactivité de l'eau ne représente plus qu'environ $10 \%$ de sa valeur initiale. Le facteur de concentration du radionucléide en fonction du poids sec, calculé à ce moment là, est de 2600 pour Platyhypnidium riparioides et 8800 pour Fontinalis antipyretica. Un tel écart paraît a priori surprenant, mais la conjonction de la différence de morphologie des deux espèces et du comportement de l'110m Ag peuvent le justifier, en grande partie au moins. En effet, pour une même biomasse, la surface présentée par les feuilles est supérieure pour Fontinalis antipyretica que pour Platyhypnidium riparioides, caractéristique particulièrement importante du fait que l'110m $\mathrm{Ag}$ s'adsorbe très rapidement sur toutes les surfaces disponibles (Garnier-Laplace, 1991). En conditions dynamiques, après 3 jours, cette différence spécifique apparaît également et exactement dans les mêmes proportions (tableau 1). Les valeurs du facteur de concentration sont inférieures à celles calculées pour la contamination statique, malgré l'accumulation d'une quantité supérieure $d^{\prime 110 m} \mathrm{Ag}$ résultant de l'apport chronique du radionucléide. Cette distorsion est liée à la définition même du facteur de concentration qui représente le rapport entre la radioactivité dans les mousses et la radioactivité dans le milieu. En conditions statiques, ce paramètre peut donc être surestimé, et dans des proportions d'autant plus grandes que la concentration du radionucléide dans l'eau sera devenue faíble, comme c'est le cas pour $\mathrm{l}^{\prime} 110 \mathrm{~m} \mathrm{Ag}$ notamment. Dans ces conditions, la valeur atteinte par le facteur de concentration n'a qu'une signification relative quant aux possibilités d'accumulation du radionucléide.

L'expérimentation en conditions dynamiques a dû être interrompue après 3 jours, en raison de la forte radioactivité consécutive à l'introduction d'une quantité élevée $d^{\prime 110 m} \mathrm{Ag}\left(1,6.10^{5} \mathrm{~Bq}\right)$, nécessaire pour compenser sa fixation par les mousses et sa forte adsorption sur tous les composants du dispositif expérimental en contact avec l'eau. Toutefois, les données obtenues tendent à indiquer que l'accumulation du radionucléide était loin d'avoir atteint son niveau maximal, même sí la dispersion des valeurs ne permet pas une extrapolation suffisamment fiable. Compte tenu de l'intérêt radioécologique que présente $\mathrm{l}^{110 \mathrm{~m}} \mathrm{Ag}$, d'autres études doivent être entreprises afin 
Tableau 1. - Données radjoécologiques relatives à l'accumulation et à l'élimination de $1^{110 \mathrm{~m}} \mathrm{Ag}$ par deux espèces de mousses dulcaquicoles.

Table 1. - Radioecological data related to ${ }^{110 \mathrm{~m}} \mathrm{Ag}$ accumulation and elimination by two species of freshwater mosses.

\begin{tabular}{lccc}
\hline & \multicolumn{2}{c}{$\begin{array}{c}\text { CONDITIONS STATIQUES } \\
\text { (1 jour de contamination) } \\
\text { Accumulation* }\end{array}$} & \multicolumn{2}{c}{\begin{tabular}{c} 
Elimination \\
\cline { 3 - 4 }
\end{tabular}} & & $\begin{array}{c}\text { \% de rétention } \\
\text { après 6 jours }\end{array}$ & $\begin{array}{l}\text { Période } \\
\text { biologique }\end{array}$ \\
\hline $\begin{array}{l}\text { Platyhypnidium } \\
\text { riparioides }\end{array}$ & $\mathrm{FC}=2600$ & $30 \%$ & $\mathrm{~Tb}=5 \mathrm{j}$ \\
$\begin{array}{l}\text { Fontinalis } \\
\text { antipyretica }\end{array}$ & $\mathrm{FC}=8800$ & $55 \%$ & $\mathrm{~Tb}=11 \mathrm{j}$ \\
\hline \hline
\end{tabular}

CONDITIONS DYNAMIQUES

(3 jours de contamination)

Accumulation

Elimination

\begin{tabular}{lccc} 
& Accumulation & \multicolumn{2}{c}{ Elimination } \\
\cline { 2 - 4 } & & $\begin{array}{l}\text { \% de rétention } \\
\text { après } 11 \text { jours }\end{array}$ & $\begin{array}{l}\text { Période } \\
\text { biologique }\end{array}$ \\
\hline $\begin{array}{l}\text { Platyhypnidium } \\
\text { riparioides }\end{array}$ & $\mathrm{FC}=1600$ & $60 \%$ & $\mathrm{~Tb}=24 \mathrm{j}$ \\
$\begin{array}{l}\text { Fontinalis } \\
\text { antipyretica }\end{array}$ & $\mathrm{FC}=5450$ & $55 \%$ & $\mathrm{~Tb}=41 \mathrm{j}$ \\
\hline
\end{tabular}

* FC calcule en fonction du poids sec

d'évaluer avec plus de précision son accumulation par les mousses dulçaquicoles, en mettant au point un protocole opératoire permettant de s'affranchir des difficultés expérimentales liées à son comportement.

Placées dans une eau non radioactive, les mousses ayant fixé de ' '110mAg se décontaminent en partie, mais ce processus est plus lent que l'accumulation. Ainsi, à la suite d'une contamination en conditions statiques, le taux de rétention du radio- nucléide après 6 jours de décontamination est de $30 \%$ pour Platyhypnidium riparioides et de $55 \%$ pour Fontinalis antipyretica. Après une contamination en conditions dynamiques, ce taux est respectivement de 60 et $70 \%$, à l'issue d'une phase de décontamination de 11 jours. La désorption de $\mathrm{l}^{110 \mathrm{~m}} \mathrm{Ag}$ par les mousses présente la même cinétique, une phase très rapide suivie d'une phase lente, pour les deux espèces et quelles que soient les conditions de la contamination. Par contre, comme 
l'indique le taux de rétention, ces deux facteurs ont une influence très marquée sur la vitesse de décontamination. Après une accumulation en conditions statiques, la période biologique correspondant à la deuxième phase de la décontamination est de 11 jours pour Fontinalis antipyretica et de 5 jours pour Platyhypnidium riparioides. Après une contamination en conditions dynamiques, les valeurs de ce paramètre sont respectivement de 41 et 24 jours pour ces deux espèces. La comparaison de l'ensemble des paramètres caractérisant les échanges du ${ }^{110 \mathrm{~m}} \mathrm{Ag}$ entre l'eau et les mousses (tableau 1) montre que, pour chacune des deux conditions, le facteur de concentration le plus élevé correspond à la période biologique la plus longue et que la valeur de celleci est d'autant plus grande que le temps d'exposition a été long. Comme cela a été indiqué précédemment il est très probable, que le phénomène d'adsorption joue un rôle prépondérant dans l'accumulation du radionucléide, mais les écarts observés pour la vitesse et le taux d'élimination suggèrent l'existence d'un processus actif, responsable de l'accumulation d'une partie du radionucléide fixé. D'après les données relatives au mécanisme d'accumulation et à la localisation des métaux accumulés par les mousses, exposées par Mouvet (1986), il est possible d'émettre l'hypothèse d'une

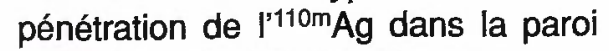
cellulaire et même dans le cytoplasme, proportionnelle au temps d'exposition. La validité de cette hypothèse reste bien sûr à démontrer par des tests plus nombreux, mais essentiellement par des études physicochimiques et histologiques.

\subsection{2. $L^{, 125} S b$}

Avec ce radionucléide, les expériences ont été menées sur une seule espèce, Platyhypnidium riparioides. Lors de la contamination en conditions statiques, et contrairement aux constatations faites pour $1^{1110 \mathrm{~m}} \mathrm{Ag}$, la concentration de l'125Sb dans l'eau est restée remarquablement stable durant les 15 jours de la phase de contamination. Cette stabilité témoigne d'une absence d'adsorption et se traduit pour les mousses par un facteur de concentration de 8 (poids sec). En raison du niveau constant de la radioactivité de l'eau, les réserves faites quant à la validité des données obtenues en conditions statiques perdent une partie de leur fondement si bien que la valeur du facteur de concentration peut être considérée comme tout à fait réaliste. Cependant, en conditions dynamiques, ce paramètre atteint une valeur plus élevée, de l'ordre de 30 après 7 jours. Compte tenu des données concernant la contamination en conditions statiques, ce résultat apparaît paradoxal, d'autant plus qu'il traduit une augmentation de la concentration de $1^{125} \mathrm{Sb}$ dans les mousses, supérieure à celle qui se produit dans l'eau en raison des ajouts répétés de radionucléide. Dans l'état actuel de nos connaissances, il est difficile d'expliquer ce paradoxe mais il est possible que le mode de contamination intervienne. Ainsi, en conditions dynamiques, le courant 
d'eau pourrait entraîner une adsorption plus forte en augmentant les contacts entre les feuilles des mousses et le radionucléide en solution. Toutefois, malgré l'intérêt que présente ce problème, d'un point de vue radioécologique la très faible accumulation de I'125Sb par Platyhypnidium riparioides est le fait essentiel à retenir. En effet cette constatation explique très bien que $\left.\right|^{1124} \mathrm{Sb}$ ne soit pas détecté dans les mousses en aval des centrales à eau pressurisée, malgré sa présence dans les effluents liquides, dont il représente en moyenne de 12 à $14 \%$ de la radioactivité totale hors tritium (EDF, 1991). Inversement, les mesures effectuées sur les mousses prélevées en aval des centres de production nucléaire valident les résultats expérimentaux. II faut donc souligner que cette association de données concernant l'antimoine constitue un exemple parfaitement significatif de la complémentarité des travaux in situ et des études en laboratoire.

La désorption du ${ }^{125} \mathrm{Sb}$ par Platyhypnidium riparioides est très peu influencée par les conditions de la contamination préalable. Après 15 jours de décontamination, le taux de rétention du radionucléide par les mousses est de l'ordre de $50 \%$ et la période biologique est de 20 jours. Compte tenu d'une période physique de 60 jours, cette vitesse d'élimination appliquée au ${ }^{124} \mathrm{Sb}$ se traduit par une période effective de 16 jours. Associée à la faible valeur du facteur de concentration, cette donnée justifie la difficulté de détection de ce radionu- cléide dans les échantillons de mousses prélevés in situ.

\subsection{3. $L e^{106} R u$}

Les expériences réalisées avec le ${ }^{106} \mathrm{Ru}$ sur Platyhypnidium riparioides représentent, d'un point de vue radioécologique, un ensemble d'études relativement complet, dans la mesure où l'influence de plusieurs facteurs a été testée (Vray, 1990). En ce qui concerne la contamination, trois conditions ont été retenues : statique, dynamique continue et dynamique intermittente. Pour la deuxième de ces conditions l'effet de la température, de l'intensité lumineuse et de la photopériode a été considéré. Toutefois, pour ne pas multiplier abusivement le nombre des expériences et afin de prendre en compte des situations écologiques réalistes, ces facteurs abiotiques ont été regroupés pour simuler 2 saisons opposées, l'été et l'hiver. Pour la première, l'eau est maintenue à une température de $12 \pm 1^{\circ} \mathrm{C}$, l'intensité lumineuse est de $20 \mu \mathrm{E} \cdot \mathrm{m}^{-2} \cdot \mathrm{s}^{-1}$ et la photopériode de $16 \mathrm{~h}$ de lumière et $8 \mathrm{~h}$ d'obscurité. Pour la saison hivernale, les valeurs de ces paramètres sont respectivement de $4 \pm 1^{\circ} \mathrm{C}, 13,5 \mu \mathrm{E} \cdot \mathrm{m}^{-2} \mathrm{~s}^{-1}$ et $8 \mathrm{~h} / 16 \mathrm{~h}$. D'autre part, afin d'essayer de mettre en évidence le rôle et l'importance des mécanismes passifs dans l'accumulation du ${ }^{106} \mathrm{Ru}$, une expérience de contamination à l'obscurité, en conditions statiques, a été réalisée. Enfin, une contamination en conditions dynamiques a été effectuée avec Fontinalis antipyretica dans le but de tester l'influence de l'es- 
pèce. Pour toutes les conditions, à la suite de la phase d'accumulation qui a duré 5 jours, la décontamination des mousses a été suivie pendant plusieurs semaines.

\section{Contamination des mousses}

Pour l'ensemble des conditions, l'évolution du facteur de concentration peut être décrite par une équation constituée de la somme de deux exponentielles (fig. 7). Dans certains cas ( $a, b, c$, et d), la valeur asymptotique est égale ou peu différente de celle calculée après 5 jours. Pour les autres (e et $f$ ), la contamination maximale ne paraît pas être atteinte à l'issue de cette période, mais l'extrapolation ne peut se faire sans réserves.

Les conditions de la contamination ont une influence très marquée sur l'accumulation du ${ }^{106} \mathrm{Ru}$ par Platyhypnidium riparioides (fig. $7 \mathrm{a}, \mathrm{b}, \mathrm{c}$ ). Comme dans le cas de $1^{110 \mathrm{~m}} \mathrm{Ag}$, et pour les mêmes raisons, le facteur de concentration le plus élevé correspond à la contamination en conditions statiques. Comme cela a été expliqué précédemment, ce résultat, s'il n'a pas une grande valeur explicative ni prédictive en ce qui concerne la contamination in situ, indique cependant que le ${ }^{106} \mathrm{Ru}$ a une forte tendance à s'adsorber sur les mousses. D'autre part, la radioactivité de l'eau restant à un niveau significatif, on peut conclure à l'établissement d'un équilibre entre fixation et désorption, tout le radionucléide disponible n'étant pas accumulé par les mousses. L'apport intermittent de radionucléide dans le milieu est la condition qui représente le mieux la situation ren- contrée en aval des installations nucléaires, en raison des cycles de rejet des effluents liquides. En conséquence, la valeur du facteur de concentration obtenu dans ce cas (environ 1300) doit être considérée comme la plus significative et retenue pour le décodage d'une contamination in situ.

Les résultats concernant l'influence de la saison sur la contamination de Platyhypnidium riparioides posent des problèmes d'interprétation, qui ne seront abordés que très succinctement ici. La comparaison des facteurs de concentration après 5 jours d'accumulation (fig. $7 \mathrm{~b}$ et $e$ ) indique une fixation plus importante en conditions estivales ( $F C=1900)$ qu'en conditions hivernales $(F C=1600)$. Par contre, la cinétique de contamination montre que, dans le premier cas, la valeur obtenue correspond à l'instauration d'un état d'équilibre, alors que dans le second, le phénomène est en phase croissante, ce qui se traduit par une valeur asymptotique plus élevée (2570). Cependant, cette extrapolation doit être considérée avec prudence, dans la mesure où l'équation établie est un simple ajustement et non pas un modèle ayant un fondement théorique. Cet exemple met en évidence les difficultés d'interpréter, dans certains cas, les résultats expérimentaux et la nécessité d'améliorer et d'approfondir les travaux in vitro. Toutefois, malgré ces réserves, le peu de différence observée entre les valeurs, permet de conclure que l'influence des saisons, telles qu'elles ont été définies dans le protocole ex- 


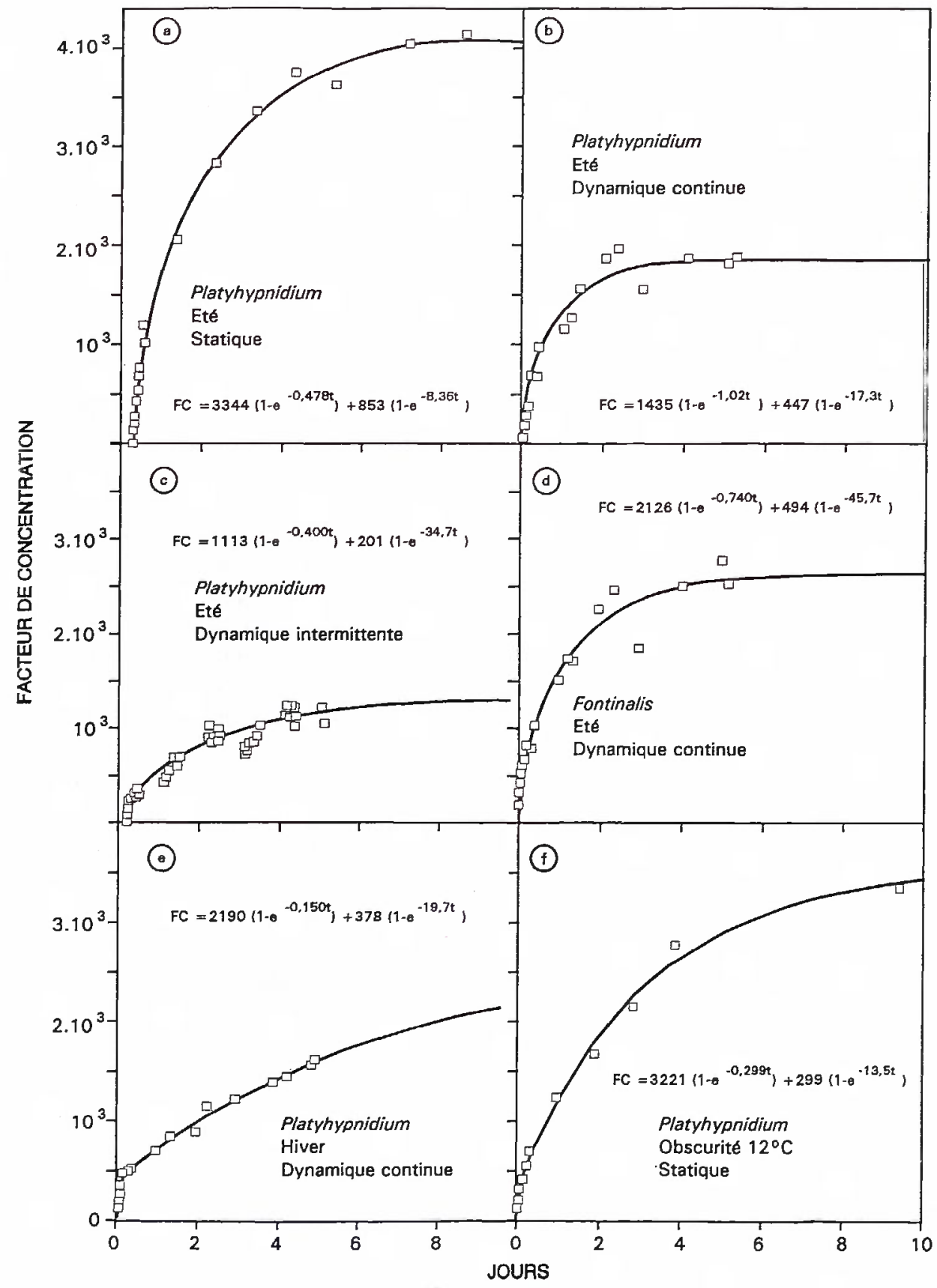

Fig. 7. - Cinétique de l'accumulation du ${ }^{106}$ Ru par des mousses dulçaquicoles en fonction des conditions de la contamination.

Fig. 7. - Kinetic of ${ }^{106} \mathrm{Ru}$ accumulation by freshwater mosses as a function of contamination conditions. 
périmental, sur l'accumulation du ${ }^{106}$ Ru par les mousses est très limitée.

L'influence de l'espèce (fig. $7 b$ et d) apparaît assez nettement, le facteur de concentration du ${ }^{106} \mathrm{Ru}$ étant significativement plus élevé pour Fontinalis antipyretica $(\mathrm{FC}=2800)$ que pour Platyhypnidium riparioides $\left(F C=1880\right.$ ). Comme pour $1^{\prime 110 m} \mathrm{Ag}$, l'écart entre les valeurs du facteur de concentration peut s'expliquer par la morphologie des mousses et le comportement du radionucléide. A cet égard, il faut souligner que la différence spécifique est étroitement liée à la capacité à s'adsorber des deux radionucléides, qui est beaucoup plus forte pour $1^{1110 \mathrm{~m}} \mathrm{Ag}$ que pour le ${ }^{106} \mathrm{Ru}$. Cette constatation constitue un argument supplémentaire dans la démonstration du caractère essentiellement passif de l'accumulation des radionucléides.

L'effet de la lumière sur l'accumulation du ${ }^{106} \mathrm{Ru}$ par Platyhypnidium riparioides est très net, que l'on considère le facteur de concentration après 5 jours de contamination ou sa valeur asymptotique (fig. $7 \mathrm{a}$ et f). Chez la même espèce, mais pour une intensité lumineuse beaucoup plus élevée $\left(157 \mu \mathrm{E} \cdot \mathrm{m}^{2} \cdot \mathrm{s}^{-1}\right)$, un effet semblable a été signalé par Mouvet (1969) pour l'accumulation du $\mathrm{Cr}^{3+}$. La comparaison des valeurs du facteur de concentration du ${ }^{106} \mathrm{Ru}$ témoigne de l'importance des phénomènes passifs dont le rôle essentiel apparaît incontestable, mais indique également l'intervention, dans une proportion significative, d'un processus lié à la lumière.

En conclusion de ce paragraphe, il faut souligner que, s'il y a des écarts entre les valeurs du facteur de concentration du ${ }^{106} \mathrm{Ru}$, ceux-ci sont relativement faibles par rapport aux variations importantes présentées par les conditions de contamination. D'autre part, pour ce radionucléide, la différence maximale est inférieure à celle constatée entre les deux espèces de mousse pour l'accumulation

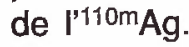

\section{Décontamination des mousses}

Alors que la cinétique d'accumulation indiquait un processus biphasique, la décontamination des mousses ne présente qu'une phase, quelles que soient les conditions de la contamination (fig. 8). Globalement, les valeurs des périodes biologiques sont homogènes et sont comprises entre 30 et 50 jours, ce qui correspond à des périodes effectives de 28 à 45 jours.

\subsection{Synthèse des données rela- tives à la contamination de Platy- hypnidium riparioides}

Dans le tableau $\mathrm{n}^{\circ} 2$ sont rassemblées les données radioécologiques tirées de l'ensemble des études expérimentales menées dans le Laboratoire de Radioécologie des Eaux Continentales sur la contamination, en conditions statiques, de Platyhypnydium riparioides par plusieurs radionucléides. Sur la base du facteur de concentration, quatre cas peuvent être distingués. Le premier regroupe 


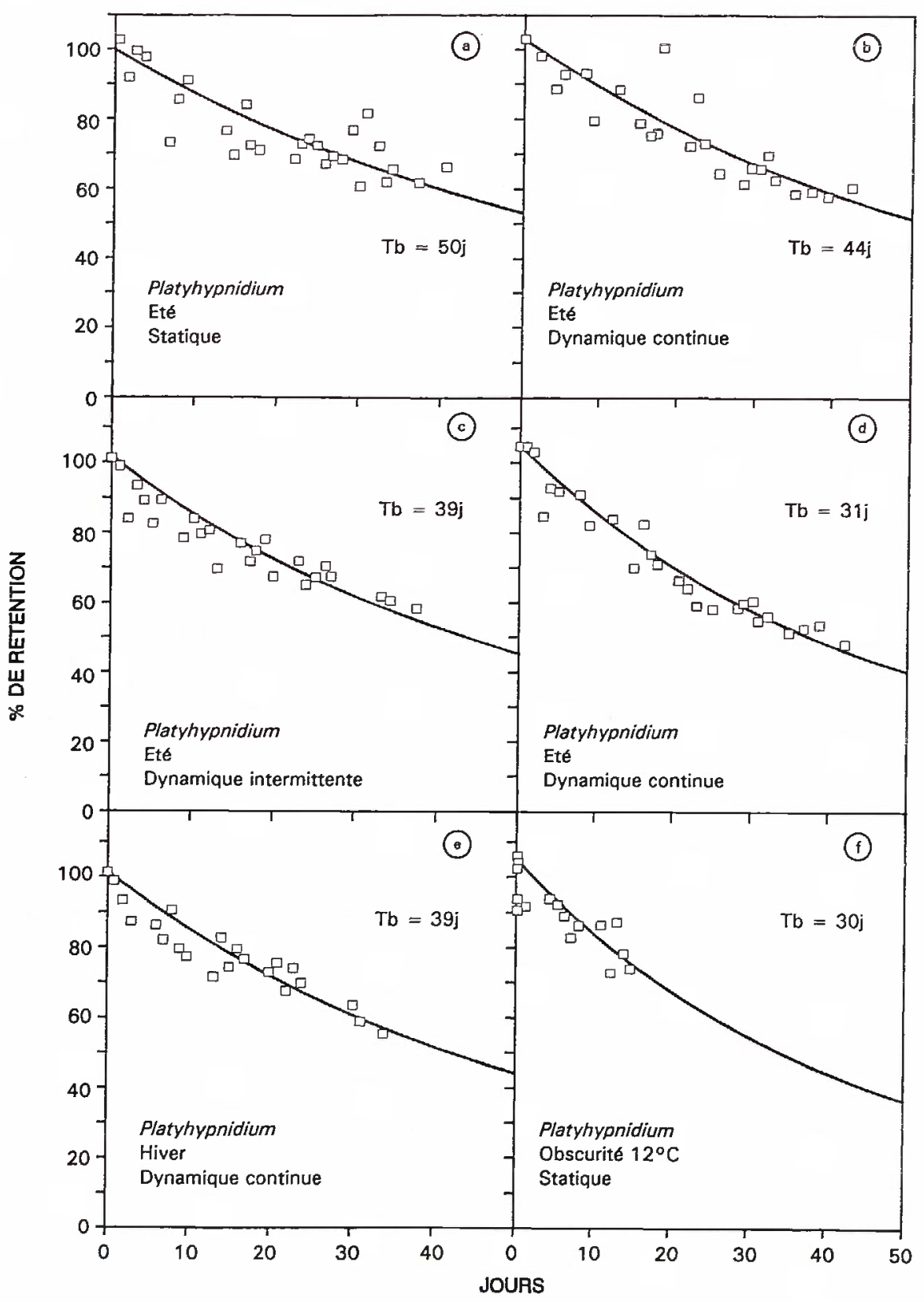

Fig. 8. - Cinétique de l'élimination du ${ }^{106}$ Ru par des mousses dulçaquicoles en fonction des conditions de la contamination préalable.

Fig. 8. - Kinetic of ${ }^{106}$ Ru elimination by freshwater mosses as a function of previous contamination conditions. 
le ${ }^{60} \mathrm{Co}$, le ${ }^{54} \mathrm{Mn}$ et le ${ }^{137} \mathrm{Cs}$ pour lesquels les valeurs sont de beaucoup les plus élevées. Elles sont probablement un peu surestimées, mais sont conformes aux niveaux d'accumulation constaté in situ, notamment pour les radiocobalt et le ${ }^{54} \mathrm{Mn}$. Le deuxième cas concerne le ${ }^{22} \mathrm{Na}$ et $\mathrm{l}^{\prime 125} \mathrm{Sb}$, dont les facteurs de concentration, qui sont peu élevés, reflètent parfaitement les possibilités réelles d'accumulation, dans la mesure où, dans les expériences, la radioactivité de l'eau n'a quasiment pas diminué. Pour le ${ }^{106} \mathrm{Ru}$, la cinétique de contamination montre, qu'après 7 jours, le facteur de concentration a atteint sa valeur maximale, bien que du radionucléide reste encore dans l'eau. Le dernier cas concerne l'110m Ag pour lequel la valeur du facteur de concentration est manifestement sous-estimée. En effet, d'une part elle ne correspond pas aux capacités d'adsorption du radionucléide et, d'autre part, après 24 heures l'état d'équilibre était loin d'être atteint.

A propos des critiques et réserves formulées quant à l'évaluation in vitro des facteurs de concentration, l'occasion nous a été donnée de pouvoir confronter certaines de nos données expérimentales aux résultats de mesures effectuées sur des mousses prélevées in situ. Lors des études réalisées sur le site de la centrale de Nogent-sur-Seine en juillet 1989, une étroite collaboration avec le DSRE et la direction de cette installation nous a permis de connaître les quantités de ${ }^{54} \mathrm{Mn},{ }^{58} \mathrm{Co},{ }^{60} \mathrm{Co}$ et ${ }^{137} \mathrm{Cs}$ rejetées depuis le mois d'avril et notamment le $1^{\text {er }}$ juillet. A l'aide des renseignements concernant le débit du fleuve ce jourlà et les caractéristiques du rejet, il a été possible de calculer la concentration théorique des 4 radionucléides dans l'eau ainsi que dans les mousses prélevées $2 \mathrm{~km}$ en aval de la centrale. Cette deuxième évaluation a été réalisée d'après les facteurs de concentration déterminés expérimentalement (tableau 2). La comparaison des deux ensembles de données, montre que les valeurs calculées ne sont pas très éloignées des résultats des mesures (tableau 3 ), et qu'ainsi les facteurs de concentration évalués in vitro, même en conditions statiques, ont une réelle signification (tableau 3). Les différences observées sont très peu importantes, compte tenu des nombreux facteurs pouvant intervenir dans la contamination des mousses par les radionucléides considérés. En premier lieu, il faut préciser que la radioactivité théorique de l'eau est calculée pour la zone immédiatement en aval du rejet alors que les mousses ont été prélevées $2 \mathrm{~km}$ plus loin. Il faut également tenir compte des effluents rejetés au cours des semaines précédentes, les quantités de radionucléides fixées pouvant se cumuler, au moins partiellement. Enfin, le phénomène de décontamination doit être pris en compte, d'autant plus que les rejets sont intermittents et que le lessivage des mousses, dû au courant, doit favoriser la désorption des radionucléides. Ces trois faits paraissent tout à fait suffisants pour expliquer les différences constatées, et en particulier la plus forte concentration du ${ }^{58} \mathrm{Co}$ et 
Tableau 2. - Données radioécologiques relatives à l'accumulation en conditions statiques et à l'élimination de divers radionucléides par Platyhypnidium riparioides.

Table 2. - Radioecological data related to accumulation in static conditions and elimination of different radionuclides by Platyhypnidium riparioides.

\begin{tabular}{|c|c|c|c|c|}
\hline \multirow[t]{2}{*}{ RADIONUCLEIDE } & \multirow{2}{*}{$\begin{array}{l}\text { Facteur de } \\
\text { concentration } \\
\text { (poids sec) }\end{array}$} & \multirow{2}{*}{$\begin{array}{l}\text { Temps } \\
\text { (jours) }\end{array}$} & \multicolumn{2}{|c|}{ Périodes biologiques } \\
\hline & & & $\mathrm{Tb}_{1}$ & $\mathrm{~Tb}_{2}$ \\
\hline${ }^{60} \mathrm{Co}$ & $20000^{(1)}$ & 3 & \multicolumn{2}{|c|}{ Indéterminées* } \\
\hline${ }^{54} \mathrm{Mn}$ & $30000^{(1)}$ & 2 & 2 jours $s^{(1)}$ & Indéterminée* \\
\hline${ }^{137} \mathrm{Cs}$ & $10000^{(1)}$ & 3 & 6 heures $^{(1)}$ & Indéterminée ${ }^{*}$ \\
\hline${ }^{22} \mathrm{Na}$ & $17^{(1)}$ & 56 & 24 heures $^{(1)}$ & Indéterminée* \\
\hline${ }^{125} \mathrm{Sb}$ & 8 & 15 & \multicolumn{2}{|c|}{20 jours } \\
\hline${ }^{110 \mathrm{~m}} \mathrm{Ag}$ & 2600 & 1 & \multicolumn{2}{|c|}{5 jours } \\
\hline${ }^{106} \mathrm{Ru}$ & 4100 & 7 & \multicolumn{2}{|c|}{50 jours } \\
\hline
\end{tabular}

(1) d'après Maurel-Kermarec et al. (1984) * Indéterminée = très longue

Tableau 3. - Exemple de confrontation de résultats expérimentaux à des mesures in situ. Table 3. - Example of comparison of experimental results with in situ measurements.

\begin{tabular}{lcccc}
\hline Radionucléides & ${ }^{54} \mathrm{Mn}$ & ${ }^{58} \mathrm{Co}$ & ${ }^{60} \mathrm{Co}$ & ${ }^{137} \mathrm{Cs}$ \\
\hline $\begin{array}{l}\text { Concentration théorique dans l'eau } \\
\text { le } 01 / 07 / 1989\left(B q . I^{-1}\right)\end{array}$ & $1,3 \cdot 10^{-3}$ & 0,12 & $4,5.10^{-3}$ & $1,5.10^{-3}$ \\
$\begin{array}{l}\text { Concentration théorique* dans les } \\
\text { mousses (Bq.kg-1sec) }\end{array}$ & 39 & 2400 & 90 & 15 \\
$\begin{array}{l}\text { Concentration mesurée dans les mousses } \\
\text { prélevées le 05/07/1989 (Bq.kg-1 }\end{array}$ & 23 & 3470 & 63 & 6 \\
\hline
\end{tabular}

* D'après les valeurs des FC du tableau 2.

les plus faibles valeurs concernant les trois autres radionucléides. A propos des deux isotopes du cobalt, il faut indiquer qu'en prenant en compte tous les effluents rejetés entre le 5 avril et le $1^{\text {er }}$ juillet 1989 , le rapport [radionucléide] mousses/quantité totale rejetée est du même ordre (179 pour le ${ }^{58} \mathrm{Co}$ et 165 pour le ${ }^{60} \mathrm{Co}$ ). Cette similitude indique clairement qu'il n'y a pas de discrimination isotopique dans l'accumulation du cobalt par les mousses. Cet exemple, comme l'ensemble des indications tirées de la confrontation des données expérimentales et de celles provenant des 
études réalisées sur le site de la centrale de Nogent-sur-Seine, démontre la complémentarité des travaux in situ et des expériences en laboratoire.

\section{CONCLUSION}

Quelle que soit l'origine de la présence de radionucléides dans le milieu aquatique continental, les mousses constituent le meilleur indicateur de contamination, par comparaison au sédiment, aux végétaux supérieurs et aux poissons. En ce qui concerne les centrales nucléaires, la concentration des radionucléides dans les mousses reflète qualitativement, et en partie quantitativement, la composition des effluents liquides. A cet égard, il faut signaler que $\left.\right|^{1124} \mathrm{Sb}$ représente une exception notable, dans la mesure où, malgré les quantités rejetées, il n'est pas détecté dans les échantillons. Cette absence est imputable à une période physique relativement courte (60 jours), mais aussi à un facteur de concentration particulièrement faible, comme l'a démontré une évaluation in vitro, avec $\mathrm{p}^{125} \mathrm{Sb}$. Cette seconde explication met en évidence l'intérêt que présente la complémentarité des études in situ et des expériences de laboratoire. A ce sujet, il faut signaler que toutes les comparaisons faites entre les deux types de données apportent des éléments de réponse au problème de l'influence de la spéciation des radionucléides sur leur accumulation par les organismes vivants. Ce facteur est difficile à maîtriser car, que ce soit in vitro ou in situ, il est quasiment impossible de connaître la forme chimique des radionucléides en raison de leur trop faible concentration molaire. Or, au même titre que les conditions expérimentales proprement dites et que les facteurs biotiques et abiotiques considérés, cette difficulté met en question la validité des résultats expérimentaux, d'autant plus que la forme chimique des radionucléides dans les solutions utilisées pour les expériences est, à l'évidence, différente de celle des effluents des centrales. Sur ce sujet précis, la confrontation des données expérimentales et des résultats des mesures in situ, et notamment les comparaisons faites à propos des études réalisées sur le site de la centrale de Nogent-sur-Seine, montre une concordance suffisamment étroite pour conclure à une validité satisfaisante des études in vitro. En conséquence, cette conclusion permet logement de déduire que la forme chimique des radionucléides n'a pas une influence majeure sur la contamination des mousses.

Il est incontestable que l'ensemble des données présentées fait apparaître des lacunes qu'il convient d'essayer de combler. A cet effet, un programme de recherche, liant étroitement études in situ et travaux expérimentaux, a été élaboré, en collaboration avec des responsables du Département Environnement Aquatique et Atmosphérique (Direction des Etudes et Recherches) d'EDF. Ainsi, une série d'expériences 
doit être entreprise avec les radionucléides majeurs des effluents liquides des centrales nucléaires selon un protocole standardisé, mis au point d'après les études précédemment réalisées et celles menées par ailleurs avec les métaux lourds. Parallèlement, des mesures seront effectuées sur de échantillons prélevés en aval de toutes les centrales nucléaires implantées sur les fleuves français, dans le cadre d'un suivi radioécologique réalisé à la demande du Département Sécurité Radioprotection Environnement d'EDF. Ces études in situ doivent se traduire par la mise en évidence systématique des relations, qualitatives et quantitatives, entre la composition des effluents liquides des installations et la contamination des mousses. La connaissance de ces relations, associée aux données expérimentales, doit conduire à une évaluation fiable des processus d'accumulation et de désorption des radionucléides qui permettra d'utiliser au mieux les mousses dulçaquicoles pour caractériser une contamination radioactive dans un cours d'eau.

\section{REMERCIEMENTS}

Les données présentées dans cet article sont tirées d'un grand nombre de résultats qui sont le fruit d'un travail collectif. En conséquence, les signataires de l'article tiennent à remercier toutes les personnes qui, à des titres divers, ont participé aux travaux in situ ou aux expériences en laboratoire et en particulier Foulquier L., Vray F., Garnier-Laplace J.,
Svadlenkova M., Descamps B., Rozet M., Etienne J.L. et Chabrier P.

\section{BIBLIOGRAPHIE}

Baudin J.P., Foulquier L. \& Bovard P., 1970. Dynamique du césium-137 ingéré avec la nourriture chez un poisson d'eau douce (Ictalurus melas). Cr. Acad. d'Agriculture, 16(10): 662-669.

Baudin-Jaulent Y. \& Descamps B., 1985. Proposition d'un test utilisant les mousses aquatiques pour le contrôle radioécologique de l'environnement. Radioprotection, 20(3) : 197-205.

Bovard P. \& Grauby A., 1967. The fixation of radionuclides from atmospheric fallout in peat-bog Sphagnum $S p$, PoIytrichum and Myriophyllum. Radioecological Concentration Processes. B. Aberg and F.P. Hungate ed. : 533-537. Pergamon Press Ltd. London: $1040 \mathrm{pp}$.

Descamps B., 1987. Etude radioécologique du complexe minier de Lodève (France) 1981-1985. Rapport CEA-R5409. C.E.N. Saclay : $98 \mathrm{pp}$.

Descamps B. \& Baudin-Jaulent Y., 1988. Evolution de l'impact radioécologique de l'accident de Tchernobyl dans trois bassins fluviaux français. Impact des accidents d'origine nucléaire sur l'environnement. IV Symposium de radioécologie, CEN Cadarache, France, 14-18 mars : C68-C80.

EDF, 1988. Environnement année 1987. Rapport d'activité, Annexe IX. Département Sécurité, Radioprotection, Environnement, Division Environnement, $57 \mathrm{pp}$.

EDF, 1989. Environnement année 1988. Rapport d'activité, Annexe IX. Département Sécurité, Radioprotection, Environnement, Division Environnement, $54 \mathrm{pp}$.

EDF, 1990. Environnement année 1989. Rapport d'activité, Annexe IX. Dépar- 
tement Sécurité, Radioprotection, Environnement, Division Environnement, $55 \mathrm{pp}$.

EDF, 1991. Environnement année 1990. Rapport d'activité, Annexe IX. Département Sécurité Radioprotection Environnement, Division Environnement, $59 \mathrm{pp}$.

Foulquier L. \& Grauby A. 1967. Etude comparée de la contamination expérimentale de trois espèces végétales par le césium-137. Congrès International sur la Radioécologie du Milieu, Toulouse 14-16 mars, Société Française de Radioprotection : 201-212.

Foulquier L. \& Hébrard J.P. 1976. Etude expérimentale de la fixation et de la décontamination du sodium-22 par une mousse dulçaquicole, Platyhypnidium riparioides (Hew.) Dix. Oecol. Plant., 11(3): 267-276.

Foulquier L., Lambrechts A. \& Pally M., 1987. Impact radioécologique d'une usine de retraitement de combustible nucléaire sur un fleuve, le Rhône. Proceedings of an International Conference on Nuclear Fuel Reprocessing and Management. Paris, August 23-27. Société Française d'Energie Nucléaire : 1063-1071.

Foulquier L. \& Baudin-Jaulent Y. 1989. Impact radioécologique de l'accident de Tchernobyl sur les écosystèmes aquatiques continentaux. Rapport XI3522/90 FR. Radioprotection-50. Commission des Communautés Européennes, D.G. XI : 222 pp.

Foulquier L., Garnier-Laplace J., Descamps B., Lambrechts A. \& Pally M., 1991. Exemples d'impact radioécologiques de centrales nucléaires sur des cours d'eau français. Hydroécologie appliquée, 3 (sous presse).

Garnier-Laplace J., 1991. Etude des mécanismes de transfert de l'argent-110m en eau douce. Conception et exploitation d'un modèle expérimental d'écosystème et d'un modèle mathématique de simulation de la distribution du radioélément au sein d'une chaîne tro- phique. Thèse de Doctorat, Univ. Montpellier 1I; Rapport CEA-R-5549: 199 pp.

Hébrard J.P., Foulquier L. \& Grauby A., 1968. Aperçu sur les modalités de la contamination d'une mousse dulçicole, Platyhypnidium riparioides (Hedw) Dix, par le césium-137 et le strontium-90. Rev. Bryol. et Lichenol., 36 (1-2) : 219242.

Hébrard J.P. \& Foulquier L., 1975. Introduction à la fixation du manganèse-54 par Platyhypnidium riparioides (Hedw) Dix. Rev. Bryol, et Lichenol., 41 (1): 35-54.

Lambrechts A., 1984. Essai de modélisation du transfert $\mathrm{du}{ }^{137} \mathrm{Cs}$ dans les compartiments d'un écosystème d'eau douce simplifié. Thèse de Doctorat d'Université, Univ. Aix-Marseille I: $181 \mathrm{pp}$.

Lambrechts A., Foulquier L. \& Pally M., 1988. Etude comparée de l'impact radioécologique des installations nucléaires et de l'accident de Tchernobyl sur le fleuve Rhône. Impact des accidents d'origine nucléaire sur l'environnement. $N^{e}$ Symposium de radioécologie, CEN Cadarache, France, 14-18 mars: C39-C50.

Maubert H. \& Descamps B. 1983. Etude radioécologique de la Seịne, site de Nogent-sur-Seine. Point zéro hydrologique, sédimentologique et hydrobiologique. CEA-IPSN-SERE, Laboratoire d'Etude de Pollution des Eaux, CEN Cadarache, France : 68 pp.

Maurel-Kermarrec A., 1984. Cinétique de la fixation et de la désorption d'un mélange de ${ }^{60} \mathrm{Co},{ }^{51} \mathrm{Cr},{ }^{137} \mathrm{Cs},{ }^{54} \mathrm{Mn}$ et ${ }^{22} \mathrm{Na}$ par une mousse (Platyhypnidium riparioides Hedw. Dix) et une hépatique (Chiloscyphus polyanthos L. Corda in Opiz) dulçaquicoles. Thèse $3^{\theta}$ Cycle, Univ. Aix-marseille III: 124 pp.

Maurel-Kermarrec A., Pally M., Foulquier L. \& Hébrard J.P., 1983. Cinétique de la fixation d'un mélange de césium 137, de chrome 51 , de cobalt 60 , de manganèse 54 et de sodium 22 par 
Platyhypnidium riparioides (Hedw) Dix. Cryptogamie, Bryol. Lichenol., 4 (4) : 299-313.

Maurel-Kermarrec A., Pally M., Foulquier L. \& Hébrard J.P., 1985. Cinétique de la fixation et de la désorption d'un mélange de cobalt 60 , de chrome 51 , de césium 137, de manganèse 54 et de sodium 22 par Chiloscyphus polyanthos (L.) Corda in Opiz. Cryptogamie, Bryol. Lichenol., 6 (4) : 359-377.

Mouvet C., 1979. Utilisation des bryophytes aquatiques pour l'étude de la pollution des cours d'eau par les métaux lourds et les radionucléides. Revue de Biologie et Ecologie méditerranéenne, 6 (3-4) : 193-204.

Mouvet C., 1986. Métaux lourds et mousses aquatiques. Synthèse méthodologique. Agence de l'eau RhinMeuse: 110 pp.
Ottavi L. \& Descamps B., 1988. Etudes radioécologiques du site de Golfech. Point zéro hydrologique, sédimentologique et hydrobiologique. CEA-IPSNSERE, Laboratoire d'Etudes d'Impacts et Laboratoire de Radioécologie des Eaux Continentales, CEN Cadarache, France : $56 \mathrm{pp}$.

Svensson G.K., 1967. The increasing ${ }^{137} \mathrm{Cs}$ level in forest moss in relation to the total ${ }^{137} \mathrm{Cs}$ fallout from 1961 through $1965 . \quad$ Radioecological Concentration Processes. B. Aberg and F.P. Hungate ed. : 539-546. Pergamon press Ltd. London : $1040 \mathrm{pp}$.

Vray F., 1990. Etude expérimentale de l'influence de divers facteurs abiotiques sur la fixation et l'élimination du ${ }^{106} \mathrm{Ru}$ par des populations de bryophytes dulçaquicoles. Diplôme d'Etudes Approfondie des Sciences de l'Evolution et Ecologie, Univ. Montpellier II, 26 pp. 